\title{
Joint State Sensing and Communication over Memoryless Multiple Access Channels
}

\author{
Mari Kobayashi ${ }^{1}$, Hassan Hamad ${ }^{1}$, Gerhard Kramer ${ }^{1}$, and Giuseppe Caire ${ }^{2}$ \\ ${ }^{1}$ Technical University of Munich, Germany \\ 2 Technical University of Berlin, Germany \\ Emails: \{mari.kobayashi, hassan.hamad, gerhard.kramer\}@tum.de, caire@tu-berlin.de
}

\begin{abstract}
A memoryless state-dependent multiple access channel (MAC) is considered where two transmitters wish to convey a respective message to a receiver while simultaneously estimating the respective channel state via generalized feedback. The scenario is motivated by a joint radar and communication system where the radar and data applications share the same bandwidth. An achievable capacity-distortion tradeoff region is derived that outperforms a resource-sharing scheme through a binary erasure MAC with binary states.
\end{abstract}

\section{INTRODUCTION}

Consider the communication setup depicted in Fig. 1. Two encoders each wish to convey a message to a decoder over a state-dependent multiple access channel (MAC) and simultaneously estimate their state sequence via generalized feedback $Z_{k, i-1}, k=1,2, i=2, \ldots, n$. For simplicity, we assume that at time $i$ the decoder has access to the state $S_{i}=\left(S_{1 i}, S_{2 i}\right)$. The above communication setup is motivated by joint radar and data communications, where radar-equipped transmitters track the state while exchanging data. Most current communication systems build on resource sharing, where the time and frequency resources are divided into either state sensing or communication.

We recently studied a single-user version of this problem in [1]. In this paper, we extend the results to two-user MACs. As in [1], the state information is available at the receiver, which is different from [2] where the state is estimated at the receiver. The main contributions of the paper are:

- an outer bound on the capacity-distortion region that builds on [3];

- an achievable rate-distortion region that builds on [4];

- numerical examples based on a binary erasure MAC.

This paper is organized as follows. Section II describes the model and presents our main results. Section III provides the outer bound and Section IV provides the achievability proof. We consider a binary erasure MAC with binary states in Section V.

\section{System Model And Main Results}

Consider the channel inputs $X_{k i} \in \mathcal{X}_{k}$, the channel outputs $Y_{i} \in \mathcal{Y}$, the feedback channel outputs $Z_{k i} \in \mathcal{Z}$, and channel state $S_{i} \in \mathcal{S}_{1} \times \mathcal{S}_{2}, k=1,2, i=1, \ldots, n$ linked by a discrete

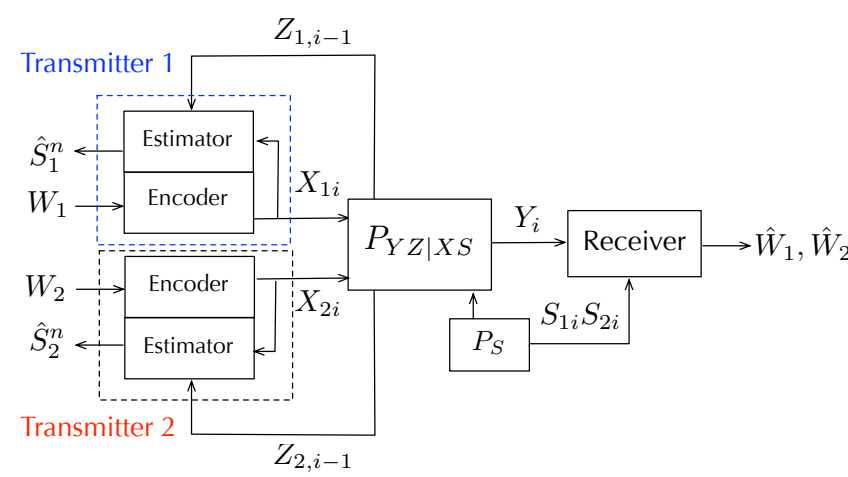

Fig. 1. State-dependent MAC with generalized feedback

memoryless channel with i.i.d. states. The joint probability distribution of these random variables can be written as

$$
\begin{gathered}
\prod_{i=1}^{n} P_{S}\left(s_{i}\right) P_{Y Z_{1} Z_{2} \mid X_{1} X_{2} S}\left(y_{i}, z_{1 i}, z_{2 i} \mid x_{1 i}, x_{2 i}, s_{i}\right) \\
P\left(x_{1 i} \mid x_{1}^{i-1}, z_{1}^{i-1}\right) P\left(x_{2 i} \mid x_{2}^{i-1}, z_{2}^{i-1}\right) .
\end{gathered}
$$

A $\left(2^{n R_{1}}, 2^{n R_{1}}, n\right)$ code for the state-dependent discrete memoryless MAC with generalized feedback consists of

- Two message sets $\mathcal{W}_{k}=\left[1: 2^{n R_{k}}\right]$ for $k=1,2$.

- Encoder $k$ : a function $\phi_{k i}: \mathcal{W}_{k} \times \mathcal{Z}_{k} \mapsto \mathcal{X}_{k}$ that assigns a symbols $x_{k i}=\phi_{k i}\left(w_{k}, z_{k}^{i-1}\right)$ for $i=1, \ldots, n$. For simplicity, we write $x_{k}^{n}=\phi_{k}^{n}\left(w_{k}, z_{k}^{n-1}\right)$ for the sequence of $n$ encoded symbols.

- Decoder: a function $g: \mathcal{Y}^{n} \times \mathcal{S}_{1}^{n} \times \mathcal{S}_{2}^{n} \mapsto \mathcal{W}_{1} \times \mathcal{W}_{2}$ that assigns a message pair $\left(\hat{w}_{1}, \hat{w}_{2}\right)=g\left(y^{n}, s^{n}\right)$.

- State estimator $k$ outputs the estimate $\hat{s}_{k}^{n}$ as a function of $x_{k}^{n}$ and $z_{k}^{n}$. We consider without loss of generality a function $\tilde{\psi}_{k}^{n}: \mathcal{X}_{k}^{n} \times \mathcal{Z}_{k}^{n} \mapsto \mathcal{S}_{k}^{n}[1$, Lemma 2] so that $\hat{s}_{k}^{n}=\tilde{\psi}_{k}^{n}\left(x_{k}^{n}, z_{k}^{n}\right)$.

The average distortion of estimator $k$ is

$$
d_{k}^{(n)}=\mathbb{E}\left[\frac{1}{n} \sum_{i=1}^{n} d_{k}\left(S_{k i}, \hat{S}_{k i}\right)\right]
$$

where $d_{k}: \mathcal{S}_{k} \times \hat{\mathcal{S}}_{k} \mapsto[0, \infty)$ measures the distortion between a state symbol and a reconstruction symbol. We consider bounded distortion functions with $d_{\max } \triangleq \max _{(k, s, \hat{s})} d_{k}(s, \hat{s})$. Let the average error probability be $P_{e}^{(n)}$. We say that 
$\left(R_{1}, R_{2}, D_{1}, D_{2}\right)$ is achievable if for all $\epsilon>0$ there is some $n$ and a $\left(2^{n R_{1}}, 2^{n R_{2}}, n\right)$ code satisfying $P_{e}^{(n)} \leq \epsilon$ and $d_{k}^{(n)} \leq D_{k}+\epsilon$ for $k=1,2$. The capacity region $\mathcal{C}\left(D_{1}, D_{2}\right)$ is the closure of achievable $\left(R_{1}, R_{2}\right)$ for specified $D_{1}, D_{2}$.

For our outer bound on $\mathcal{C}\left(D_{1}, D_{2}\right)$, we consider idealized transmitter estimators $\hat{s}_{k}=\psi_{k}^{*}\left(x_{1}, x_{2}, z_{1}, z_{2}\right), k=1,2$, that are aware of $x_{1}, x_{2}$ as well as $z_{1}, z_{2}$. The best such estimators are

$$
\begin{aligned}
& \psi_{k}^{*}\left(x_{1}, x_{2}, z_{1}, z_{2}\right)=\arg \min _{\psi_{k}: \mathcal{X}_{1} \times \mathcal{X}_{2} \times \mathcal{Z}_{1} \times \mathcal{Z}_{2} \mapsto \mathcal{S}_{k}} \\
& \sum_{s_{k} \in \mathcal{S}_{k}} P_{S_{k} \mid X_{1} X_{2} Z_{1} Z_{2}}\left(s_{k} \mid x_{1} x_{2} z_{1} z_{2}\right) d_{k}\left(s_{k}, \psi_{k}\left(x_{1}, x_{2}, z_{1}, z_{2}\right)\right)
\end{aligned}
$$

for $k=1,2$ with the conditional distortions

$c_{k}\left(x_{1}, x_{2}\right)=\mathbb{E}\left[d_{k}\left(s_{k}, \psi_{k}^{*}\left(x_{1}, x_{2}, z_{1}, z_{2}\right)\right) \mid X_{1}=x_{1}, X_{2}=x_{2}\right]$.

The following outer bound extends a bound from [3] to statedependent MACs with distortion constraints.

Theorem 1. $\mathcal{C}\left(D_{1}, D_{2}\right)$ is a subset of the union of $\left(R_{1}, R_{2}\right)$ satisfying

$$
\begin{aligned}
R_{1} & \leq I\left(X_{1} ; Y Z_{1} Z_{2} \mid S X_{2} T\right) \\
R_{2} & \leq I\left(X_{2} ; Y Z_{1} Z_{2} \mid S X_{1} T\right) \\
R_{1}+R_{2} & \leq I\left(X_{1} X_{2} ; Y Z_{1} Z_{2} \mid S T\right) \\
R_{1}+R_{2} & \leq I\left(X_{1} X_{2} ; Y \mid S\right)
\end{aligned}
$$

where $T-S X_{1} X_{2}-Y Z_{1} Z_{2}$ forms a Markov chain, and we have the dependence balance constraint

$$
I\left(X_{1} ; X_{2} \mid T\right) \leq I\left(X_{1} ; X_{2} \mid Z_{1} Z_{2} T\right)
$$

and the average distortion constraints

$$
\mathbb{E}\left[c_{k}\left(X_{1}, X_{2}\right)\right] \leq D_{k}, \quad k=1,2 .
$$

It suffices to consider $T$ whose alphabet $\mathcal{T}$ has cardinality $|\mathcal{T}| \leq 7$ (see Appendix B).

Remark 1. The result yields a number of special cases studied in the literature. Without distortion constraints and states, the bounds reduce to the ones derived in [3]. For a single user, i.e., $X_{2}$ and $Z_{2}$ constants, Theorem 1 yields the capacity-distortion tradeoff in [1]. For a special case when the feedback is output feedback $Z_{1}=Z_{2}=Y$ and we have no distortion constraints, the region reduces to [5, Section VII].

For our achievable region, we consider an estimator $\underline{\psi}_{1}^{*}\left(x_{1}, v_{2}, z_{1}\right)$ given by

$$
\begin{aligned}
& \underline{\psi}_{1}^{*}\left(x_{1}, v_{2}, z_{1}\right)=\arg \min _{\psi_{1}: \mathcal{X}_{1} \times \mathcal{V}_{2} \times \mathcal{Z}_{1} \mapsto \mathcal{S}_{1}} \\
& \sum_{s_{1} \in \mathcal{S}_{1}} P_{S_{1} \mid X_{1} V_{2} Z_{k}}\left(s_{k} \mid x_{1} v_{2} z_{k}\right) d_{1}\left(s_{1}, \psi_{1}\left(x_{1}, v_{2}, z_{k}\right)\right)
\end{aligned}
$$

yielding the estimation cost as

$$
\underline{c}_{1}\left(x_{1}, v_{2}\right)=\mathbb{E}\left[d_{1}\left(s_{1}, \underline{\psi}_{1}^{*}\left(x_{1}, v_{2}, z_{1}\right)\right) \mid X_{1}=x_{1} V_{2}=v_{2}\right]
$$

We define $\underline{\psi}_{2}^{*}\left(v_{1}, x_{2}, z_{2}\right)$ and $\underline{c}_{2}\left(v_{1}, x_{2}\right)$ similarly. The following achievable region is based on [4].

Theorem 2. $\mathcal{C}\left(D_{1}, D_{2}\right)$ includes the $\left(R_{1}, R_{2}\right)$ satisfying

$$
\begin{aligned}
R_{1} & \leq I\left(X_{1} ; Y \mid X_{2} V_{1} U S\right)+I\left(V_{1} ; Z_{2} \mid X_{2} U\right) \\
R_{2} & \leq I\left(X_{2} ; Y \mid X_{1} V_{2} U S\right)+I\left(V_{2} ; Z_{1} \mid X_{1} U\right) \\
R_{1}+R_{2} & \leq \min \left\{I\left(X_{1} X_{2} ; Y \mid S\right), I\left(X_{1} X_{2} ; Y \mid S V_{1} V_{2} U\right)\right. \\
& \left.+I\left(V_{1} ; Z_{2} \mid X_{2} U\right)+I\left(V_{2} ; Z_{1} \mid X_{1} U\right)\right\}
\end{aligned}
$$

where $V_{1} X_{1}-U-V_{2} X_{2}$ and $U V_{1} V_{2}-X_{1} X_{2}-Y Z_{1} Z_{2}$ form Markov chains, and where

$$
\begin{aligned}
& \mathbb{E}\left[\underline{c}_{1}\left(X_{1}, V_{2}\right)\right] \leq D_{1} \\
& \mathbb{E}\left[\underline{c}_{2}\left(V_{1}, X_{2}\right)\right] \leq D_{2} .
\end{aligned}
$$

\section{CONVERSE}

This section provides a sketch of proof for Theorem 1 . Details are provided in Appendix A. By following the same steps as [3] and [5], we have

$$
\begin{aligned}
& n R_{1} \leq \sum_{i=1}^{n} I\left(X_{1 i} ; Y_{i} Z_{i} \mid S_{i} X_{2 i} Z^{i-1}\right)+n \epsilon \\
& n R_{2} \leq \sum_{i=1}^{n} I\left(X_{2 i} ; Y_{i} Z_{i} \mid S_{i} X_{1 i} Z^{i-1}\right)+n \epsilon \\
& n\left(R_{1}+R_{2}\right) \leq \sum_{i=1}^{n} I\left(X_{1 i} X_{2 i} ; Y_{i} Z_{i} \mid S_{i} Z^{i-1}\right)+n \epsilon \\
& \sum_{i=1}^{n} I\left(X_{1 i} ; X_{2 i} \mid Z_{i} Z^{i-1}\right) \leq \sum_{i=1}^{n} I\left(X_{1 i} ; X_{2 i} \mid Z^{i-1}\right) .
\end{aligned}
$$

where we let $Z_{i}=\left(Z_{1 i}, Z_{2 i}\right)$. Next, suppose a genie gives both inputs $X_{1, i}, X_{2, i}$ to both transmitters when estimating $S_{k, i}$ for $j \neq k$. We then have the distortion constraints

$$
\frac{1}{n} \sum_{i=1}^{n} \mathbb{E}\left[c_{k}\left(X_{1 i}, X_{2 i}\right)\right] \leq D_{k}+\epsilon, \quad k=1,2 .
$$

Let $Q$ be uniform over $1,2, \ldots, n$ and independent of all other random variables. Define $T=\left(Q, Z_{1}^{Q-1}, Z_{2}^{Q-1}\right), X_{1 Q}=X_{1}$, and similarly for all other variables. By letting $n \rightarrow \infty$, we readily obtain $(5 a),(5 b),(5 c),(6)$ and $(7)$, while (5d) follows from the cut set bound.

\section{ACHIEVABILITY}

We use block Markov encoding and backward decoding [4]. Encoder $k$ sends $2(B-1)$ i.i.d. messages $\left\{w_{k 1}(b), w_{k 2}(b)\right\}_{b=1}^{B-1}$ over $n=B N$ channel uses. The messages $w_{k 1}(b) \in\left[1,2^{N R_{k 1}}\right]$ and $w_{k 2}(b) \in\left[1,2^{N R_{k 2}}\right], k=1,2$, $b=1, \ldots, B-1$, are uniformly distributed and mutually independent. By letting $B \rightarrow \infty$, we obtain $R_{j k} \frac{B-1}{B} \rightarrow R_{j k}$ for any $j, k=1,2$. Encoder 1 's message $w_{12}$ is decoded by encoder 2 , while encoder 2's message $w_{21}$ is decoded by encoder 1 thanks to generalized feedback, yielding encoder cooperation. 
a) Codebook Generation: Fix a pmf $P_{U}(u) \prod_{k=1}^{2} P_{V_{k} \mid U}\left(v_{k} \mid u\right) P_{X_{k} \mid V_{k} U}\left(x_{k} \mid v_{k}, u\right)$ and functions $\underline{\psi}_{1}^{*}\left(x_{1}, v_{2}, z_{1}\right), \underline{\psi}_{2}^{*}\left(v_{1}, x_{2}, z_{2}\right)$ such that the distortion constraints are satisfied. For each block $b=1, \ldots, B$, we proceed as follows:

- Generate $2^{N\left(R_{12}+R_{21}\right)}$ sequences $u^{N}\left(j_{b-1}, k_{b-1}\right)$, $j_{b-1}=1, \ldots, 2^{N R_{12}}, k_{b-1}=1, \ldots, 2^{N R_{21}}$, each according to $\prod_{i=1}^{N} P_{U}\left(u_{i}\right)$.

- For each $\left(j_{b-1}, k_{b-1}\right)$, generate $2^{N R_{12}}$ sequences $v_{1}^{N}\left(j_{b-1}, k_{b-1}, j_{b}^{\prime}\right), j_{b}^{\prime}=1, \ldots, 2^{N R_{12}}$, each according to $\prod_{i=1}^{N} P_{V_{1} \mid U}\left(v_{1 i} \mid u_{i}\left(j_{b-1}, k_{b-1}\right)\right)$. Similarly generate $v_{2}^{N}\left(j_{b-1}, k_{b-1}, k_{b}^{\prime}\right), k_{b}^{\prime}=1, \ldots, 2^{N R_{21}}$.

- For each $\left(j_{b-1}, k_{b-1}, j_{b}^{\prime}\right)$, generate $2^{N R_{11}}$ sequences $x_{1}^{N}\left(j_{b-1}, k_{b-1}, j_{b}^{\prime}, l_{b}\right), l_{b}=1, \ldots, 2^{N R_{11}}$, each according to $\prod_{i=1}^{N} P_{X_{1} \mid U V_{1}}\left(x_{1 i} \mid u_{i}\left(j_{b-1}, k_{b-1}\right), v_{1 i}\left(j_{b}^{\prime}\right)\right)$. Similarly generate $x_{2}^{N}\left(j_{b-1}, k_{b-1}, k_{b}^{\prime}, m_{b}\right), m_{b}=1, \ldots, 2^{N R_{22}}$.

b) Encoding: We set $j_{0}=k_{0}=1$ and $l_{B}=m_{B}=1$. At the end of block $b$, encoder 1 finds an index $k_{b}^{\prime}$ such that

$$
\left(u^{N}(\cdot, \cdot) v_{1}^{N}\left(\cdot, \cdot, j_{b}^{\prime}\right), v_{2}^{N}\left(\cdot, \cdot, k_{b}^{\prime}\right), x_{1}^{N}\left(\cdot, \cdot, j_{b}^{\prime}, l_{b}\right), z_{1}^{N}(b)\right) \in \mathcal{T}_{\epsilon}^{N}
$$

where the first two arguments of each variable are $j_{b-1}, k_{b-1}{ }^{1}$. Using this estimate $k_{b}^{\prime}$ from block $b$, encoder 1 transmits $x_{1}^{N}\left(j_{b}, k_{b}^{\prime}, j_{b+1}^{\prime}, l_{b+1}\right)$ in block $b+1$. Similarly, encoder 2 finds an index $j_{b}^{\prime}$ such that

$$
\left(u^{N}(\cdot, \cdot), v_{1}^{N}\left(\cdot, \cdot, j_{b}^{\prime}\right), v_{2}^{N}\left(\cdot, \cdot, k_{b}^{\prime}\right), x_{2}^{N}\left(\cdot, \cdot, k_{b}^{\prime}, m_{b}\right), z_{2}^{N}(b)\right) \in \mathcal{T}_{\epsilon}^{N} .
$$

Using the estimate $j_{b}^{\prime}$ from block $b$, encoder 2 transmits $x_{2}^{N}\left(j_{b}^{\prime}, k_{b}, k_{b+1}^{\prime}, m_{b+1}\right)$ in block $b+1$. Both encoders repeat the same procedure for each $b$.

c) Decoding: Assuming that $\left(j_{b}^{\prime}, k_{b}^{\prime}\right)$ is decoded correctly in block $b+1$, the decoder finds $\left(j_{b-1}, k_{b-1}, l_{b}, m_{b}\right)$ in block $b$ such that $u^{N}\left(j_{b-1}, k_{b-1}\right)$, $v_{1}^{N}\left(j_{b-1}, k_{b-1}, j_{b}^{\prime}\right), v_{2}^{N}\left(j_{b-1}, k_{b-1}, k_{b}^{\prime}\right), x_{1}^{N}\left(j_{b-1}, k_{b-1}, j_{b}^{\prime}, l_{b}\right)$, $x_{2}^{N}\left(j_{b-1}, k_{b-1}, k_{b}^{\prime}, m_{b}\right), s^{N}(b), y^{N}(b)$ are jointly typical. The decoder repeats this step for blocks $B$ to 1 .

d) State Estimation: For each block $b=1, \ldots, B$, encoder 1 puts out

$\hat{s}_{1}^{N}(b)=\underline{\psi}_{1}^{*}\left(x_{1}^{N}\left(j_{b-1}, k_{b-1}, j_{b}^{\prime}, l_{b}\right), v_{2}^{N}\left(j_{b-1}, k_{b-1}, k_{b}^{\prime}\right), z_{1}^{N}(b)\right)$

where $k_{b}^{\prime}$ is decoded at the end of block $b$ during encoding process. Similarly, encoder 2 lets

$\hat{s}_{2}^{N}(b)=\underline{\psi}_{2}^{*}\left(v_{1}^{N}\left(j_{b-1}, k_{b-1}, j_{b}^{\prime}\right), x_{2}^{N}\left(j_{b-1}, k_{b-1}, k_{b}^{\prime}, m_{b}\right), z_{1}^{N}(b)\right.$

where $j_{b}^{\prime}$ is known to encoder 2 from its encoding process.

1 If there is more than one such index, we select one of these indices uniformly at random. If there is no such index, we choose an index from $\left\{1, \ldots, 2^{N R_{21}}\right\}$ uniformly at random. A similar procedure applies to decoding and shall be omitted. e) Error Probability: Following the same steps as [4], we can prove that by letting $N \rightarrow \infty, P_{e}^{(n)} \rightarrow 0$ if the following conditions hold:

$$
\begin{aligned}
R_{12} & \leq I\left(V_{1} ; Z_{2} \mid X_{2} U\right) \\
R_{21} & \leq I\left(V_{2} ; Z_{1} \mid X_{1} U\right) \\
R_{11} & \leq I\left(X_{1} ; Y \mid S X_{2} V_{1} U\right) \\
R_{22} & \leq I\left(X_{2} ; Y \mid S X_{1} V_{2} U\right) \\
R_{11}+R_{22} & \leq I\left(X_{1} X_{2} ; Y \mid S V_{1} V_{2} U\right) \\
R_{12}+R_{21}+R_{11}+R_{22} & \leq I\left(X_{1} X_{2} ; Y \mid S\right)
\end{aligned}
$$

The analysis details are provided in Appendix C. Applying Fourier-Motzkin elimination, we obtain the desired expressions.

f) Distortion: If there is no decoding error, $\left(u^{N}(b)\right.$, $\left.v_{1}^{N}(b), \quad v_{2}^{N}(b), \quad x_{1}^{N}(b), \quad x_{2}^{N}(b), \quad y^{N}(b), \quad s(b)\right)$ are jointly typical for all $b$. We simplify notation and let $w_{k}=$ $\left(w_{k}(1), \ldots, w_{k}(B-1)\right)$ with $\left|\mathcal{W}_{k}\right|=2^{N(B-1)\left(R_{k 1}+R_{k 2}\right)}$ for $k=1,2$, where $w_{k}(b)$ denotes $\left(w_{k 1}(b), w_{k 2}(b)\right)$. For a given message pair $\left(w_{1}, w_{2}\right)$, we bound the average distortion for encoder 1.

$$
\begin{aligned}
d_{1}^{(n)}\left(w_{1}, w_{2}\right) \leq & P_{e}^{(n)}\left(w_{1}, w_{2}\right) d_{\max } \\
& +\left(1-P_{e}^{(n)}\left(w_{1}, w_{2}\right)\right)(1+\epsilon) \mathbb{E}\left[\underline{c}_{1}\left(X_{1}, V_{2}\right)\right]
\end{aligned}
$$

By averaging over all possible message pairs, we obtain the desired result. The details of the proof are provided in Appendix D.

\section{EXAMPLE}

Consider a MAC where the state and channel inputs are binary, $S_{k}, X_{k} \in\{0,1\}$ and the channel output is ternary:

$$
Y=S_{1} X_{1}+S_{2} X_{2}
$$

Consider Hamming distance, i.e., $d(s, \hat{s})=s \oplus \hat{s}$. For simplicity, we consider output feedback $Z_{1}=Z_{2}=Y$ and assume that $S_{1}$ and $S_{2}$ are i.i.d. Bernoulli with parameter $p_{s} \triangleq \operatorname{Pr}(S=1)$. If $p_{s}=1$, then this channel reduces to the binary erasure MAC with feedback, whose capacity region is the Cover-Leung region [6], [7] (see also [8, Chapter 17]).

We compute the optimal estimation cost. The best estimator gives either zero distortion or $\eta=\min \left\{p_{s}, 1-p_{s}\right\}$ yielding the following cost for encoder 1 (see Appendix E):

$$
\begin{aligned}
& c_{1}(0,0)=\eta P_{Y}(0), \quad c_{1}(1,1)=\eta P_{Y}(1) \\
& c_{1}(0,1)=\eta\left(P_{Y}(0)+P_{Y}(1)\right), \quad c_{1}(1,0)=0
\end{aligned}
$$

\section{A. Proposed Scheme}

We characterize an achievable tradeoff between the sum rate and the symmetric distortion of our proposed scheme.

$$
X_{k}=V_{k} \oplus \Theta_{k}=U \oplus \Sigma_{k} \oplus \Theta_{k}, \quad k=1,2
$$

where $U, \Sigma_{1}, \Sigma_{2}, \Theta_{1}, \Theta_{2}$ are mutually independent. For the sake of simplicity, we focus on the symmetric rate $R_{1}=R_{2}$ and let $U, \Sigma_{k}, \Theta_{k}$ is Bernoulli distributed with parameter $p, q, r$, respectively, for $k=1,2$. 
a) Unconstrained sum rate: We first characterize the unconstrained sum rate without distortion constraints, denoted by $R_{\text {sum-prop }}(\infty)$.

Corollary 1. The unconstrained sum rate is given by:

$$
R_{\text {sum-prop }}(\infty)=\max _{(p, q, r)} \min \left\{f_{1}(p, q, r), f_{2}(p, q, r)\right\}
$$

with $f_{1}=f_{1 a}+2\left\{f_{1 b}-f_{1 c}\right\}$, where $f_{1 a}, f_{1 b}, f_{1 c}, f_{2}$ are defined in (21) by letting $\kappa=q r+\bar{q} \bar{r}$ and $\bar{\kappa}=1-\kappa$.

The proof is provided in Appendix G.

Remark 2. For a special case of the erasure MAC with $p_{s}=$ 1 , the functions $f_{1}, f_{2}$ simplifies into:

$$
\begin{aligned}
& f_{1}(p, q, r)=H_{3}\left(r^{2}, 2 r \bar{r}, \bar{r}^{2}\right)+2\left(H_{2}(\kappa)-H_{2}(r)\right) \\
& f_{2}(p, q, r)=H_{3}\left(\bar{p} \kappa^{2}+p \bar{\kappa}^{2}, 2 \kappa \bar{\kappa}, p \kappa^{2}+\bar{p} \bar{\kappa}^{2}\right)
\end{aligned}
$$

It readily follows that $f_{2}$ is maximized by letting $p=1 / 2$, yielding $H_{2}(2 \kappa \bar{\kappa})+\kappa^{2}+\bar{\kappa}^{2}$. It can be proved that the sum rate is given by choosing $r=0$, yielding

$$
R_{\text {sum-prop }}(\infty)=\max _{q} \min \left\{2 H_{2}(q), H_{2}(2 q \bar{q})+q^{2}+\bar{q}^{2}\right\} .
$$

By choosing $q^{*}=0.2377$, the sum capacity of 1.5822 bit/channel use is achieved [7].

b) Minimum distortion: The minimum distortion $D_{\min }$ can be obtained by solving the following optimization problem.

$$
\min _{p, q, q} \sum_{\left(x_{1}, x_{2}\right)} P_{X_{1}, V_{2}}\left(x_{1}, v_{2}\right) \underline{c}_{1}\left(x_{1}, v_{2}\right)
$$

where by letting $\eta_{x}=\min \left\{p_{s} x, 1-p_{s} x\right\}$ the cost function $\underline{c}_{1}\left(x_{1}, v_{2}\right)$ is given by (see Appendix F).

$$
\begin{aligned}
& \underline{c}_{1}(0,0)=\eta P_{Y}(0)+\eta_{r} P_{Y}(1), \quad \underline{c}_{1}(1,1)=P_{Y}(1) \eta_{\bar{r}} \\
& \underline{c}_{1}(0,1)=\eta\left(P_{Y}(0)+P_{Y}(1)\right), \quad \underline{c}_{1}(1,0)=P_{Y}(1) \eta_{r}
\end{aligned}
$$

The solution of (22) is achieved by choosing $X_{1}=X_{2}=U$, yielding zero sum rate. With this choice $(q=r=0)$, the estimation cost coincides with the idealized one. Intermediate points between the unconstrained sum rate and the minimum distortion can be evaluated by the parametrized optimization similarly to the single-user case [1].

\section{B. Resource-Sharing}

We consider a resource sharing scheme that uses feedback only for state estimation purpose. Then, we can achieve $\left(D_{\min }, 0\right)$. The other extreme point is the unconstrained sum rate point without feedback. After some straightforward computation, we obtain:

$$
\begin{aligned}
R_{\text {sum-no-fb }}(\infty) & =\max _{P_{Q} P_{X_{1} \mid Q} P_{X_{2} \mid Q}} H(Y \mid S Q) \\
& =\max _{a} 2 p_{s} \bar{p}_{s} H_{2}(a)+p_{s}^{2} H_{3}\left(a^{2}, 2 a \bar{a}, \bar{a}^{2}\right) \\
& =2 p_{s} \bar{p}_{s}+\frac{3 p_{s}^{2}}{2}
\end{aligned}
$$

where the last equality holds by choosing $a=\frac{1}{2}$. The corresponding distortion is given by a fixed estimator independent of feedback. Namely, we consider $\hat{s}_{k}=0$ if $p_{s}<\frac{1}{2}$ and $\hat{s}_{k}=1$ if $p_{s} \geq \frac{1}{2}$. This yields the distortion of $\eta=\min \left\{p_{s}, 1-p_{s}\right\}$. In summary, the resource sharing scheme achieves any tradeoff between $\left(D_{\min }, 0\right)$ and $\left(\eta, R_{\mathrm{sum}-\mathrm{no}-\mathrm{fb}}\right)$.

\section{Outer Bound}

By applying the upper bound (1) to the binary erasure MAC with binary states, we have

$$
\begin{aligned}
R_{k} & \leq H\left(X_{k} \mid S X_{j} T\right), \forall k=1,2, \forall j \neq k \\
R_{1}+R_{2} & \leq H(Y \mid S T) \leq H(Y)
\end{aligned}
$$

We apply the technique used in [7] to the state-dependent erasure MAC. By focusing on the symmetric rate, we define $p_{t} \triangleq \operatorname{Pr}(T=t), a_{t} \triangleq \operatorname{Pr}\left(X_{k}=1 \mid T=t\right)$ for $k=1,2$. By noticing that $H\left(Y \mid\left(s_{1}, s_{2}\right), X_{2} T\right)$ is positive only for $\left(s_{1}, s_{2}\right)=(1,0),(11)$ and $H\left(Y \mid\left(s_{1}, s_{2}\right), X_{1} T\right)$ is positive only for $\left(s_{1}, s_{2}\right)=(01),(11)$, it readily follows that

$$
\begin{aligned}
H\left(Y \mid S X_{2} T\right) & =H\left(Y \mid S X_{1} T\right)=p_{s} \sum_{t} p_{t} H_{2}\left(a_{t}\right) \\
& =p_{s} H_{2}\left(\phi\left(2 \sum_{t} p_{t} a_{t} \bar{a}_{t}\right)\right)
\end{aligned}
$$

$$
\begin{aligned}
f_{1 a} & =2 \bar{p}_{s} p_{s} H_{2}(r)+p_{s}^{2} H_{3}\left(r^{2}, 2 r \bar{r}, \bar{r}^{2}\right) \\
f_{1 b} & =-\bar{p} \kappa\left[\left(\bar{p}_{s}+p_{s} \kappa\right) \log \left(\bar{p}_{s}+p_{s} \kappa\right)+\left(p_{s} \bar{\kappa}\right) \log \left(p_{s} \bar{\kappa}\right)\right] \\
& -p \bar{\kappa}\left[\left(\bar{p}_{s}+p_{s} \bar{\kappa}\right) \log \left(\bar{p}_{s}+p_{s} \bar{\kappa}\right)+\left(p_{s} \kappa\right) \log \left(p_{s} \kappa\right)\right] \\
& -\bar{p} \bar{\kappa}\left[\left(\bar{p}_{s}^{2}+p_{s} \bar{p}_{s} \kappa\right) \log \left(\bar{p}_{s}^{2}+p_{s} \bar{p}_{s} \kappa\right)+\left(\bar{p}_{s} p_{s}+p_{s} \bar{p}_{s} \bar{\kappa}+p_{s}^{2} \kappa\right) \log \left(\bar{p}_{s} p_{s}+p_{s} \bar{p}_{s} \bar{\kappa}+p_{s}^{2} \kappa\right)+\left(p_{s}^{2} \bar{\kappa}\right) \log \left(p_{s}^{2} \bar{\kappa}\right)\right] \\
& -p \kappa\left[\left(\bar{p}_{s}^{2}+p_{s} \bar{p}_{s} \bar{\kappa}\right) \log \left(\bar{p}_{s}^{2}+p_{s} \bar{p}_{s} \bar{\kappa}\right)+\left(\bar{p}_{s} p_{s}+p_{s} \bar{p}_{s} \kappa+p_{s}^{2} \bar{\kappa}\right) \log \left(\bar{p}_{s} p_{s}+p_{s} \bar{p}_{s} \kappa+p_{s}^{2} \bar{\kappa}\right)+\left(p_{s}^{2} \kappa\right) \log \left(p_{s}^{2} \kappa\right)\right] \\
f_{1 c} & =-(\bar{p} \bar{q} \kappa+p q \bar{\kappa})\left[\left(\bar{p}_{s}+p_{s} \bar{r}\right) \log \left(\bar{p}_{s}+p_{s} \bar{r}\right)+p_{s} r \log \left(p_{s} r\right)\right] \\
& -(\bar{p} q \kappa+p \bar{q} \bar{\kappa})\left[\left(\bar{p}_{s}+p_{s} r\right) \log \left(\bar{p}_{s}+p_{s} r\right)+p_{s} \bar{r} \log \left(p_{s} \bar{r}\right)\right] \\
& -(\bar{p} \bar{q} \bar{\kappa}+p q \kappa)\left[\left(\bar{p}_{s}^{2}+p_{s} \bar{p}_{s} \bar{r}\right) \log \left(\bar{p}_{s}^{2}+p_{s} \bar{p}_{s} \bar{r}\right)+\left(p_{s} \bar{p}_{s}+p_{s} \bar{p}_{s} r+p_{s}^{2} \bar{r}\right) \log \left(p_{s} \bar{p}_{s}+p_{s} \bar{p}_{s} r+\bar{r}\right)+\left(p_{s}^{2} r\right) \log \left(p_{s}^{2} r\right)\right] \\
& -(\bar{p} q \bar{\kappa}+p \bar{q} \kappa)\left[\left(\bar{p}_{s}^{2}+p_{s} \bar{p}_{s} r\right) \log \left(\bar{p}_{s}^{2}+p_{s} \bar{p}_{s} r\right)+\left(p_{s} \bar{p}_{s}+p_{s} \bar{p}_{s} \bar{r}+p_{s}^{2} r\right) \log \left(p_{s} \bar{p}_{s}+p_{s} \bar{p}_{s} \bar{r}^{2}+p_{s}^{2} r\right)+\left(p_{s}^{2} \bar{r}\right) \log \left(p_{s}^{2} \bar{r}\right)\right] \\
f_{2} & =2 p_{s} \bar{p}_{s} H_{2}(p \kappa+\bar{p} \bar{\kappa})+p_{s}^{2} H_{3}\left(\bar{p} \kappa^{2}+p \bar{\kappa}^{2}, 2 \kappa \bar{\kappa}, p \kappa^{2}+\bar{p}^{2}\right)
\end{aligned}
$$




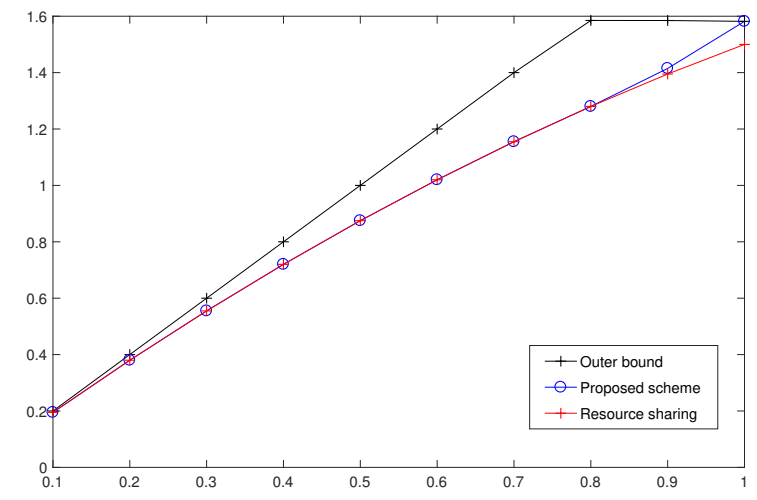

Fig. 2. Unconstrained sum rate vs. state probability $p_{s}$.

where we defined a function $\phi(t)=\frac{1}{2}(1-\sqrt{1-2 t})$ for $t \in$ $[0,1 / 2]$ and used the concavity of $H_{2}(\phi(t))$. We also have

$$
\begin{aligned}
H(Y)= & H_{3}\left(\bar{p}_{s}^{2}+2 p_{s} \bar{p}_{s} \sum_{t} p_{t} \bar{a}_{t}+p_{s}^{2} \sum_{t} p_{t} \bar{a}_{t}^{2},\right. \\
& \left.2 p_{s} \bar{p}_{s} \sum_{t} p_{t} a_{t}+2 p_{s}^{2} \sum_{t} p_{t} a_{t} \bar{a}_{t}, p_{s}^{2} \sum_{t} p_{t} a_{t}^{2}\right) \\
\leq & H_{2}\left(2 p_{s} \bar{p}_{s} \sum_{t} p_{t} a_{t}+2 p_{s}^{2} \sum_{t} p_{t} a_{t} \bar{a}_{t}\right) \\
& -\left[2 p_{s} \bar{p}_{s} \sum_{t} p_{t} a_{t}+p_{s}^{2} \sum_{t} p_{t}\left(2 a_{t} \bar{a}_{t}\right)\right]
\end{aligned}
$$

where the last inequality follows from $H_{3}(a, b, c)=$ $\frac{H_{3}(a, b, c)+H_{3}(c, b, a)}{2} \leq H_{2}(b)+1-b$, By noticing that the bounds in (25) and (26) depend only on two parameters $\alpha=2 \sum_{t} p_{t} a_{t} \bar{a}_{t}$ and $\gamma=\sum_{t} p_{t} a_{t}$, we readily obtain

$$
\begin{gathered}
R_{\text {sum-out }}(\infty)=\max _{\alpha, \gamma} \min \left\{2 p_{s} H_{2}(\phi(\alpha)), H_{2}\left(2 p_{s} \bar{p}_{s} \gamma+p_{s}^{2} \alpha\right)\right. \\
\left.\quad+1-\left(2 p_{s} \bar{p}_{s} \gamma+p_{s}^{2} \alpha\right)\right\} \\
=\max _{\beta, \gamma} \min \left\{2 p_{s} H_{2}(\beta), H_{2}\left(2 p_{s} \bar{p}_{s} \gamma+2 p_{s}^{2} \beta \bar{\beta}\right)\right. \\
\left.\quad+1-\left(2 p_{s} \bar{p}_{s} \gamma+2 p_{s}^{2} \beta \bar{\beta}\right)\right\} .
\end{gathered}
$$

where the last equality follows by letting $\beta=\phi(\alpha)$, or equivalently $\alpha=2 \beta \bar{\beta}$. The minimum distortion can be calculated similarly to (22) by replacing the estimation cost $\underline{c}_{1}\left(x_{1}, v_{2}\right)$ with the idealized estimation cost $c_{1}\left(x_{1}, x_{2}\right)$.

\section{Numerical Result}

Fig. 2 shows the unconstrained sum rate performance as a function of the state probability $p_{s}$. For the case of $p_{s}=1$, the sum capacity is $1.5822 \mathrm{bit} / \mathrm{channel}$ use. The proposed scheme yields a visible gain with respect to the resource-sharing for $p_{s}>0.8$ when feedback becomes useful for the unconstrained sum rate. The outer bound is not very tight for $p_{s}$ closed to one. Fig. 3 shows the tradeoff between the sum rate and the symmetric distortion for $p_{s}=0.7$. The proposed scheme achieves a significant gain compared to the resource sharing

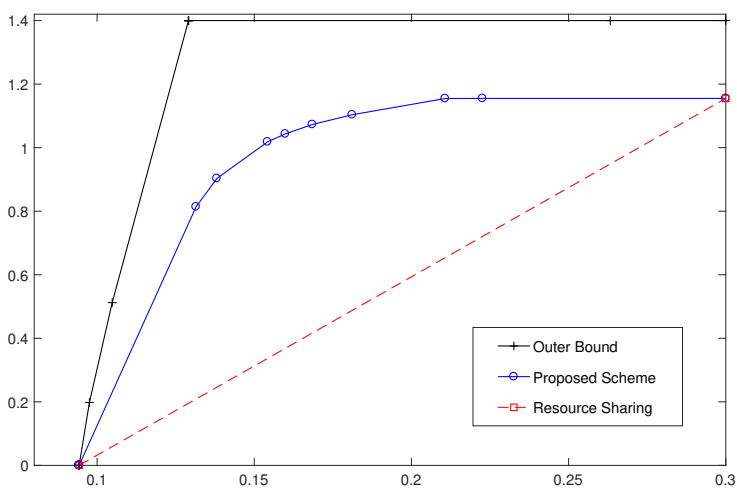

Fig. 3. Tradeoff between sum rate and distortion for $p_{s}=0.7$.

scheme in terms of tradeoff. Moreover, the proposed scheme achieves near-optimal performance for small distortion values.

Although restricted to a very simple setup, the current work demonstrates a high potential of joint sensing and communication, that exploits feedback both for state sensing and communication.

\section{REFERENCES}

[1] M. Kobayashi, G. Caire, and G. Kramer, "Joint State Sensing and Communication: Optimal Tradeoff for a Memoryless Case," in 2018 IEEE Int. Symp. Inf. Theory, Vail, CO, June 17-22, 2018., June, 2018.

[2] W. Zhang, W. Vedantam, and U. Mitra, "Joint Transmission and State Estimation: A Constrained Channel Coding Approach," IEEE Trans. Info. Theory, vol. 57, no. 10, pp. 7084-7095, 2011.

[3] R. Tandon and S. Ulukus, "Dependence balance based outer bounds for Gaussian networks with cooperation and feedback,' IEEE Trans. Info. Theory, vol. 57, no. 7, pp. 4063-4086, 2011.

[4] F. Willems, "Information Theoretical Results for the Discrete Memoryless Multiple Access Channel," Ph. D. thesis, Katholieke Universiteit Leuven, Belgium, 1989.

[5] A. P. Hekstra and F. Willems, "Dependence balance bounds for singleoutput two-way channels," IEEE Trans. Info. Theory, vol. 35, no. 1, pp. 44-53, 1989.

[6] T Cover and C Leung, "An achievable rate region for the multiple-access channel with feedback," IEEE Trans. Info. Theory, vol. 27, no. 3, pp. 292-298, 1981.

[7] F. Willems, "The feedback capacity region of a class of discrete memoryless multiple access channels (Corresp.)," IEEE Trans. Info. Theory, vol. 28, no. 1, pp. 93-95, 1982.

[8] A. El Gamal and Y.-H. Kim, Network Information Theory, Cambridge University Press, 2011. 
APPENDIX A

Proof OF THEOREM 1

First we derive single-user bounds. As above, we define $Z=\left(Z_{1}, Z_{2}\right) \in \mathcal{Z}$, and bound

$$
\begin{aligned}
n R_{1}= & H\left(W_{1}\right)=H\left(W_{1} \mid W_{2}\right) \\
= & I\left(W_{1} ; Y^{n} S^{n} Z^{n} \mid W_{2}\right)+H\left(W_{1} \mid W_{2} Y^{n} S^{n} Z^{n}\right) \\
& \stackrel{(a)}{\leq} I\left(W_{1} ; Y^{n} S^{n} Z^{n} \mid W_{2}\right)+n \epsilon \\
= & \sum_{i=1}^{n} I\left(W_{1} ; Y_{i} S_{i} Z_{i} \mid W_{2} Y^{i-1} S^{i-1} Z^{i-1}\right)+n \epsilon \\
= & \sum_{i=1}^{n} H\left(Y_{i} S_{i} Z_{i} \mid W_{2} Y^{i-1} S^{i-1} Z^{i-1}\right) \\
& -H\left(Y_{i} S_{i} Z_{i} \mid W_{1} W_{2} Y^{i-1} S^{i-1} Z^{i-1}\right)+n \epsilon \\
& \stackrel{(b)}{=} \sum_{i=1}^{n} H\left(Y_{i} S_{i} Z_{i} \mid W_{2} X_{2 i} Y^{i-1} S^{i-1} Z^{i-1}\right) \\
& -H\left(Y_{i} S_{i} Z_{i} \mid W_{1} W_{2} X_{1 i} X_{2 i} Y^{i-1} S^{i-1} Z^{i-1}\right)+n \epsilon \\
& \stackrel{(c)}{\leq} \sum_{i=1}^{n} H\left(Y_{i} S_{i} Z_{i} \mid X_{2 i} Z^{i-1}\right) \\
& -H\left(Y_{i} S_{i} Z_{i} \mid W_{1} W_{2} X_{1 i} X_{2 i} Y^{i-1} S^{i-1} Z^{i-1}\right)+n \epsilon \\
& \stackrel{(d)}{=} \sum_{i=1}^{n} H\left(Y_{i} S_{i} Z_{i} \mid X_{2 i} Z^{i-1}\right) \\
& -H\left(Y_{i} S_{i} Z_{i} \mid X_{1 i} X_{2 i} Z^{i-1}\right)+n \epsilon \\
= & \sum_{i=1}^{n} I\left(X_{1 i} ; Y_{i} S_{i} Z_{i} \mid X_{2 i} Z^{i-1}\right)+n \epsilon \\
& \left.-2^{i}\right)
\end{aligned}
$$

where (a) follows from Fano's inequality; (b) follows by applying the encoding function in both terms; (c) follows by removing conditioning on $W_{2}, Y^{i-1}, S^{i-1}$ in the first term; (d) follows from the Markov chain $\left(W_{1}, W_{2}, Y^{i-1}, S^{i-1}\right)-$ $\left(X_{1 i}, X_{2 i}, Z^{i-1}\right)-\left(Y_{i}, S_{i}, Z_{i}\right)$. Following similar steps, we obtain also

$$
\begin{array}{r}
n R_{2} \leq \sum_{i=1}^{n} I\left(X_{2 i} ; Y_{i} S_{i} Z_{i} \mid X_{1 i} Z^{i-1}\right)+n \epsilon \\
n\left(R_{1}+R_{2}\right) \leq \sum_{i=1}^{n} I\left(X_{1 i} X_{2 i} ; Y_{i} S_{i} Z_{i} \mid Z^{i-1}\right)+n \epsilon
\end{array}
$$

The proof of the dependence balance constraint follows the same steps as [5]. We start from

$$
\begin{aligned}
0 & \leq I\left(W_{1} ; W_{2} \mid Z^{n}\right) \\
& \stackrel{(a)}{=} I\left(W_{1} ; W_{2} \mid Z^{n}\right)-I\left(W_{1} ; W_{2}\right) \\
& \stackrel{(b)}{=}-I_{3}\left(W_{1} ; W_{2} ; Z^{n}\right) \\
& =-\sum_{i=1}^{n} I_{3}\left(W_{1} ; W_{2} ; Z_{i} \mid Z^{i-1}\right) \\
& \stackrel{(c)}{=}-\sum_{i=1}^{n}\left[H\left(Z_{i} \mid Z^{i-1}\right)-H\left(Z_{i} \mid W_{1} Z^{i-1}\right)\right. \\
& \left.-H\left(Z_{i} \mid W_{2} Z^{i-1}\right)+H\left(Z_{i} \mid W_{1} W_{2} Z^{i-1}\right)\right] \\
& \stackrel{(d)}{=}-\sum_{i=1}^{n}\left[H\left(Z_{i} \mid Z^{i-1}\right)-H\left(Z_{i} \mid X_{1 i} W_{1} Z^{i-1}\right)\right. \\
& \left.-H\left(Z_{i} \mid X_{2 i} W_{2} Z^{i-1}\right)+H\left(Z_{i} \mid X_{1 i} X_{2 i} W_{1} W_{2} Z^{i-1}\right)\right] \\
& \stackrel{(e)}{=}-\sum_{i=1}^{n}\left[H\left(Z_{i} \mid Z^{i-1}\right)-H\left(Z_{i} \mid X_{1 i}\right)\right. \\
& \left.-H\left(Z_{i} \mid X_{2 i}\right)+H\left(Z_{i} \mid X_{1 i} X_{2 i}\right)\right] \\
& =\sum_{i=1}^{n}-I_{3}\left(X_{1 i} ; X_{2 i} ; Z_{i} \mid Z^{i-1}\right) \\
& \stackrel{(f)}{=} \sum_{i=1}^{n} I\left(X_{1 i} ; X_{2 i} \mid Z_{i} Z^{i-1}\right)-I\left(X_{1 i} ; X_{2 i} \mid Z^{i-1}\right)
\end{aligned}
$$

where (a) follows because $W_{1}$ and $W_{2}$ are independent; (b) follows from the definition $I_{3}(A ; B ; C)=I(A ; B)-$ $I(A ; B \mid C)$; (c) follows from the definition $I_{3}(A ; B ; C)=$ $H(A)+H(B)+H(C)-H(A B)-H(A C)-H(B C)+$ $H(A B C)$; (d) follows by applying the encoding functions in last three terms; (e) follows from the Markov chain $\left(W_{1}, W_{2}, Z^{i-1}\right)-\left(X_{1 i}, X_{2 i}\right)-Z_{i}$; (f) follows from the same recursive expression used in (b).

Now, we study the distortion constraints. From the definition, a $\left(2^{n R_{1}}, 2^{n R_{2}}, n\right)$ code must satisfy for $k=1,2$

$$
\begin{aligned}
& \frac{1}{n\left|\mathcal{W}_{1}\right|\left|\mathcal{W}_{2}\right|} \sum_{\left(w_{1}, w_{2}\right)} \sum_{i=1}^{n} \\
& \mathbb{E}\left[\mathbb{E}\left[d_{k}\left(S_{k, i}, \hat{S}_{k, i}\right) \mid X_{k}^{n}=\phi_{k}^{n}\left(w_{k}, Z_{k}^{n}\right), \forall k\right]\right] \leq D_{k}+\epsilon_{n}
\end{aligned}
$$

where the outer expectation is w.r.t. $Z^{n}$ while the inner expectation is w.r.t. $S_{k}^{n}$ conditioned on $Z^{n}, w_{1}, w_{2}$. Notice that $\hat{S}_{k, i}$ is a deterministic function of $X_{k}^{n}, Z_{k}^{n}$. Assuming that a genie provides the other encoder's input $X_{j i}$ and $Z_{j}^{n}$ to encoder $k$ when estimating $S_{k i}$ for $j \neq k$, the LHS of (31) can be written as:

$$
\begin{aligned}
& \frac{1}{n\left|\mathcal{W}_{1}\right|\left|\mathcal{W}_{2}\right|} \sum_{\left(w_{1}, w_{2}\right)} \sum_{i=1}^{n} \\
& \min _{\psi_{k}^{\prime}: \mathcal{X}_{1} \times \mathcal{X}_{2} \times \mathcal{Z}^{n_{\mapsto} \mapsto \mathcal{S}_{k}}} \mathbb{E}\left[d_{k}\left(S_{k, i}, \psi_{k}^{\prime}\left(X_{1 i}, X_{2 i}, Z^{n}\right)\right) \mid X_{1 i} X_{2 i}\right]
\end{aligned}
$$


where the expectation is w.r.t. the joint distribution of $S_{k}^{n}, Z^{n}$ conditioned on $X_{1 i}, X_{2 i}$. In order to proceed further, we use the following useful lemma.

Lemma 1. [2, Lemma 1] For three arbitrary random variables $U \in \mathcal{U}, V \in \mathcal{V}, J \in \mathcal{J}$, satisfying the Markov chain $U-V-J$, and for an arbitrary function $d: \mathcal{U} \times \mathcal{U} \mapsto \mathbb{R}$, we have

$$
\min _{f: \mathcal{V} \mapsto \mathcal{U}} \mathbb{E}[d(U, f(V))]=\min _{g: \mathcal{V} \times \mathcal{J} \mapsto \mathcal{U}} \mathbb{E}[d(U, g(V, J))]
$$

We apply this lemma by letting $U=S_{k i}, V=$ $\left(X_{1 i}, X_{2 i}, Z_{1 i}, Z_{2 i}\right)$, and $J=\left(\left\{Z_{l}\right\}_{l \neq i}\right)$ and noticing

$$
S_{k i}-X_{1 i}, X_{2 i}, Z_{i}-\left\{Z_{l}\right\}_{l \neq i},
$$

forms a Markov chain, we have

$$
\begin{aligned}
& \min _{\psi_{k}^{\prime}: \mathcal{X}_{1} \times \mathcal{X}_{2} \times \mathcal{Z}_{1}^{n} \mathcal{Z}^{n} \mathcal{S}_{k}} \mathbb{E}\left[d_{k}\left(S_{k, i}, \psi_{k}^{\prime}\left(X_{1 i}, X_{2 i}, Z^{n}\right)\right) \mid X_{1 i} X_{2 i}\right] \\
& =\min _{\psi_{k}: \mathcal{X}_{1} \times \mathcal{X}_{2} \times \mathcal{Z}_{k} \mapsto \mathcal{S}_{k}} \mathbb{E}\left[d_{k}\left(S_{k i}, \psi_{k}\left(X_{1 i}, X_{2 i}, Z_{i}\right) \mid X_{1 i} X_{2 i}\right]\right. \\
& =c_{k}\left(x_{1 i}, x_{2 i}\right)
\end{aligned}
$$

where the last equality is from the definition (4). This further simplifies the distortion constraints into for $k=1,2$

$$
\frac{1}{n\left|\mathcal{W}_{1}\right|\left|\mathcal{W}_{2}\right|} \sum_{\left(w_{1}, w_{2}\right) \in \mathcal{W}_{1} \times \mathcal{W}_{2}} \sum_{i=1}^{n} c_{k}\left(x_{1 i}, x_{2 i}\right) \leq D_{k}+\epsilon_{n},
$$

Notice that the empirical input distribution $P_{X_{1 i}, X_{2 i}}\left(x_{1}, x_{2}\right)$ is induced by the uniformly selected message pair, i.e. $\left(X_{1 i}\left(w_{1}\right), X_{2 i}\left(w_{2}\right)\right)$ with probability $\frac{1}{\left|\mathcal{W}_{1}\right| \mathcal{W}_{2} \mid}$ for every pair $\left(w_{1}, w_{2}\right) \in \mathcal{W}_{1} \times \mathcal{W}_{2}$. Hence, for a sufficiently large $n$, the conditions (35) reduce to for $k=1,2$

$$
\frac{1}{n} \sum_{i=1}^{n} \sum_{\left(x_{1}, x_{2}\right) \in \mathcal{X}_{1} \times \mathcal{X}_{2}} P_{X_{1 i} X_{2 i}}\left(x_{1}, x_{2}\right) c_{k}\left(x_{1}, x_{2}\right) \leq D_{k}+\epsilon_{n}
$$

\section{Combining bounds}

We will combine the multi-letter upper bounds (27), (28) and (29) together with the multi-letter constraints (30) and (36). To this end, we introduce a uniformly distributed random variable $Q \in[1, n]$ independent of all other variables as well as an auxiliary random variable $T=\left(Q, Z^{Q-1}\right)$. By letting $X_{1 Q}=$ $X_{1}, X_{2 Q}=X_{2}, Y_{Q}=Y, Z_{Q}=Z$ and letting $n \rightarrow \infty$, we readily obtain

$$
\begin{aligned}
R_{1} & \leq I\left(X_{1} ; Y S Z \mid X_{2} T\right) \\
R_{2} & \leq I\left(X_{2} ; Y S Z \mid X_{1} T\right) \\
R_{1}+R_{2} & \leq I\left(X_{1} X_{2} ; Y S Z \mid T\right) \\
0 & \leq I\left(X_{1} ; X_{2} \mid Z T\right)-I\left(X_{1} ; X_{2} \mid T\right) .
\end{aligned}
$$

(5d) follows from the cut set bound

$$
R_{1}+R_{2} \leq I\left(X_{1} X_{2} ; Y S\right)=I\left(X_{1} X_{2} ; Y \mid S\right) .
$$

As $n \rightarrow \infty$, we have also

$$
\mathbb{E}\left[c_{k}\left(x_{1}, x_{2}\right)\right] \leq D_{k} .
$$

This establishes the converse proof.

\section{APPENDIX B}

Proof of CARdinality Constraint on $T$

First we remark that the dependence balance constraint (6), i.e. $I\left(X_{1} ; X_{2} \mid T\right) \leq I\left(X_{1} ; X_{2} \mid Z T\right)$, can be rewritten as

$$
I\left(X_{1} X_{2} ; Z \mid T\right) \leq I\left(X_{1} ; Z \mid X_{2} T\right)+I\left(X_{2} ; Z \mid X_{1} T\right) .
$$

We can see this easily

$$
\begin{aligned}
I\left(X_{1} X_{2} ; Z \mid T\right) & =I\left(X_{1} ; Z \mid X_{2} T\right)+I\left(X_{2} ; Z \mid T\right) \\
& \leq I\left(X_{1} ; Z \mid X_{2} T\right)+I\left(X_{2} ; Z \mid X_{1} T\right)
\end{aligned}
$$

where the last inequality follows because

$$
\begin{aligned}
I\left(X_{2} ; Z \mid X_{1} T\right) & =I\left(X_{2} ; X_{1} Z \mid T\right)-I\left(X_{1} ; X_{2} \mid T\right) \\
& \geq I\left(X_{2} ; X_{1} Z \mid T\right)-I\left(X_{2} ; X_{1} \mid Z T\right) \\
& =I\left(X_{2} ; Z \mid T\right) .
\end{aligned}
$$

Let $\mathcal{P}_{k}$ be a subset of pmfs on $\mathcal{X}_{k}$ and let $p\left(x_{1}, x_{2} \mid t\right) \in$ $\mathcal{P} \triangleq \mathcal{P}_{1} \times \mathcal{P}_{2}$, indexed by $t \in \mathcal{T}$ for an arbitrary set $\mathcal{T}$, be a collection of conditional pmfs on $\mathcal{X}_{1} \times \mathcal{X}_{2}$. Consider the following functions that map an element of $\mathcal{P}$ into an element of $\mathbb{R}$

$$
\begin{aligned}
& g_{1}\left(P_{X_{1}, X_{2}}\right) \triangleq I\left(X_{1} ; Y Z \mid S X_{2}\right) \\
& g_{2}\left(P_{X_{1}, X_{2}}\right) \triangleq I\left(X_{2} ; Y Z \mid S X_{1}\right) \\
& g_{3}\left(P_{X_{1}, X_{2}}\right) \triangleq I\left(X_{1} X_{2} ; Y Z \mid S\right) \\
& g_{4}\left(P_{X_{1}, X_{2}}\right) \triangleq I\left(X_{1} X_{2} ; Y \mid S\right) \\
& g_{5}\left(P_{X_{1}, X_{2}}\right) \triangleq I\left(X_{1} X_{2} ; Z\right) \\
& g_{6}\left(P_{X_{1}, X_{2}}\right) \triangleq I\left(X_{1} ; Z \mid X_{2}\right) \\
& g_{7}\left(P_{X_{1}, X_{2}}\right) \triangleq I\left(X_{2} ; Z \mid X_{1}\right)
\end{aligned}
$$

By using the support Lemma [8], we find

$$
\begin{aligned}
I\left(X_{1} ; Y Z \mid S X_{2} T\right) & =\int_{\mathcal{T}} g_{1}\left(p\left(x_{1}, x_{2} \mid t\right)\right) d F(t) \\
& =\int_{\mathcal{T}} I\left(X_{1} ; Y Z \mid S X_{2} T=t\right) d F(t) \\
& =\sum_{t^{\prime}} I\left(X_{1} ; Y Z \mid S X_{2} T^{\prime}=t^{\prime}\right) p\left(t^{\prime}\right)
\end{aligned}
$$

Similarly, we can express all other mutual informations in terms of $T^{\prime}$. Now we have shown that considering only random variables $T^{\prime}$ with constraint $\left|\mathcal{T}^{\prime}\right| \leq 7$ preserves all the quantities in our outer bound. This establishes the proof of the cardinality bound.

\section{APPENDIX C \\ ANALYSIS OF ERROR PROBABILITY}

We define the following events in (44). $\mathcal{E}_{1}\left(k_{b}^{\prime}\right)$ and $\mathcal{E}_{2}\left(j_{b}^{\prime}\right)$ are the events corresponding to (14) and (15) such that encoders 1 and 2 find a jointly typical index $k_{b}^{\prime}, j_{b}^{\prime}$ at the end of block $b$, respectively. Next, we consider the decoding events separately for $b=B, b=B, \ldots, 2$, and $b=1$. By recalling that there are no more fresh messages to send in block $B$, i.e. $j_{B}^{\prime}=l_{B}=1$ and $k_{B}^{\prime}=m_{B}=1$, we let $\mathcal{E}_{3}\left(j_{B-1}, k_{B-1}\right)$ 
denote the event that the decoder finds jointly typical indices $\left(j_{B-1}, k_{B-1}\right)$, where we let $\tilde{y}=(y, s)$ denote the augmented channel output including the state. For $b=2, \ldots, B$, assuming that the decoding of $\left(j_{b}^{\prime}, k_{b}^{\prime}\right)$ is done successfully in block $b+1$, we define the event $\mathcal{E}_{4}\left(j_{b-1}, k_{b-1}, l_{b}, m_{b}\right)$. Finally, for $b=1$ since we have $j_{0}=k_{0}=1$, we define the event $\mathcal{E}_{5}\left(l_{1}, m_{1}\right)$. We can now bound the average error probability as

$$
\begin{aligned}
P_{e}^{(n)} & \leq \operatorname{Pr}\left\{\bigcup _ { b = 1 } ^ { B - 1 } \left(\overline{\mathcal{E}}_{1}\left(k_{b}^{\prime}\right) \bigcup\left(\cup_{k \neq k_{b}^{\prime}} \mathcal{E}_{1}(k)\right)\right.\right. \\
& \bigcup \bigcup_{b=1}^{B-1}\left(\overline{\mathcal{E}}_{2}\left(j_{b}^{\prime}\right) \bigcup\left(\cup_{j \neq j_{b}^{\prime}} \mathcal{E}_{2}(j)\right)\right) \\
& \bigcup \overline{\mathcal{E}}_{3}\left(j_{B-1}, k_{B-1}\right) \bigcup\left(\cup_{(j, k) \neq\left(j_{B-1}, k_{B-1}\right)} \mathcal{E}_{3}(j, k)\right) \\
& \bigcup \bigcup_{b=2}^{B-1}\left(\overline{\mathcal{E}}_{4}\left(j_{b-1}, k_{b-1}, l_{b}, m_{b}\right)\right. \\
& \left.\bigcup\left(\cup{ }_{\left(j_{b-1}, k_{b-1}, l_{b}, m_{b}\right)}^{(j, k, l, m) \neq} \mathcal{E}_{4}(j, k, l, m)\right)\right) \\
& \left.\bigcup \overline{\mathcal{E}}_{5}\left(l_{1}, m_{1}\right) \bigcup\left(\cup_{(l, m) \neq\left(l_{1}, m_{1}\right)} \mathcal{E}_{5}(l, m)\right)\right\}
\end{aligned}
$$

By considering all possible error events and then applying the union bound, we obtain

$$
\begin{aligned}
P_{e}^{(n)} \leq & \sum_{b=1}^{B-1} \operatorname{Pr}\left\{\overline{\mathcal{E}}_{1}\left(k_{b}^{\prime}\right)\right\}+\sum_{b=1}^{B-1} \sum_{k \neq k_{b}^{\prime}} \operatorname{Pr}\left\{\mathcal{E}_{1}(k)\right\}+\sum_{b=1}^{B-1} \operatorname{Pr}\left\{\overline{\mathcal{E}}_{2}\left(j_{b}^{\prime}\right)\right\} \\
& \left.+\sum_{b=1}^{B-1} \sum_{j \neq j_{b}^{\prime}} \operatorname{Pr}\left\{\mathcal{E}_{2}(j)\right)\right\}+\operatorname{Pr}\left\{\overline{\mathcal{E}}_{3}\left(j_{B-1}, k_{B-1}\right)\right\} \\
& +\sum_{\substack{(j, k) \neq \\
\left(j_{B}-1, k_{B-1}\right)}} \operatorname{Pr}\left\{\mathcal{E}_{3}(j, k)\right\}+\sum_{b=2}^{B-1} \operatorname{Pr}\left\{\overline{\mathcal{E}}_{4}\left(j_{b-1}, k_{b-1}, l_{b}, m_{b}\right)\right\} \\
& +\sum_{b=2}^{B-1} \sum_{\substack{(j, k, l, m) \neq \\
\left(j_{b-1}, k_{b-1}, l_{b}, m_{b}\right)}} \operatorname{Pr}\left\{\mathcal{E}_{4}(j, k, l, m)\right\} \\
& +\operatorname{Pr}\left\{\overline{\mathcal{E}}_{5}\left(l_{1}, m_{1}\right)\right\}+\sum_{(l, m) \neq\left(l_{1}, m_{1}\right)} \operatorname{Pr}\left\{\mathcal{E}_{5}(l, m)\right\}
\end{aligned}
$$

In order to further simplify the upper bound on the error probability, we assume without loss of generality that $k_{b}^{\prime}=$ $j_{b}^{\prime}=1$ for $b=1, \ldots, B-1, j_{b-1}=k_{b-1}=1$ for $b=$ $2, \ldots, B$, and $l_{b}=m_{b}=1$ for $b=1, \ldots, B-1$.

$$
\begin{aligned}
P_{e}^{(n)} & \leq(B-1) \operatorname{Pr}\left\{\overline{\mathcal{E}}_{1}(1)\right\}+(B-1) \sum_{k \neq 1} \operatorname{Pr}\left\{\mathcal{E}_{1}(k)\right\} \\
& \left.+(B-1) \operatorname{Pr}\left\{\overline{\mathcal{E}}_{2}(1)\right\}+(B-1) \sum_{j \neq 1} \operatorname{Pr}\left\{\mathcal{E}_{2}(j)\right)\right\} \\
& +\operatorname{Pr}\left\{\overline{\mathcal{E}}_{3}(1,1)\right\}+\sum_{(j, k) \neq(1,1)} \operatorname{Pr}\left\{\mathcal{E}_{3}(j, k)\right\} \\
& +(B-2) \operatorname{Pr}\left\{\overline{\mathcal{E}}_{4}(1,1,1,1)\right\}+(B-2) \sum_{\substack{(j, k, l, m) \\
\neq(1,1,1,1)}} \operatorname{Pr}\left\{\mathcal{E}_{4}(j, k, l, m)\right\} \\
& +\operatorname{Pr}\left\{\overline{\mathcal{E}}_{5}(1,1)\right\}+\sum_{(l, m) \neq(1,1)} \operatorname{Pr}\left\{\mathcal{E}_{5}(l, m)\right\}
\end{aligned}
$$

where we can further express

$$
\begin{aligned}
& \quad \sum_{(j, k, l, m) \neq(1,1,1,1)} \operatorname{Pr}\left\{\mathcal{E}_{4}(j, k, l, m)\right\} \\
& =\sum_{(j, k) \neq(1,1)} \sum_{(l, m)} \operatorname{Pr}\left\{\mathcal{E}_{4}(j, k, l, m)\right\}+\sum_{l \neq 1, m \neq 1} \operatorname{Pr}\left\{\mathcal{E}_{4}(1,1, l, m)\right\} \\
& +\sum_{l \neq 1} \operatorname{Pr}\left\{\mathcal{E}_{4}(1,1, l, 1)\right\}+\sum_{m \neq 1} \operatorname{Pr}\left\{\mathcal{E}_{4}(1,1,1, m)\right\}
\end{aligned}
$$

By the law of large numbers, as $N \rightarrow \infty$, we obtain $\operatorname{Pr}\left\{\overline{\mathcal{E}}_{1}(1)\right\} \rightarrow 0$. The same holds for $\operatorname{Pr}\left\{\overline{\mathcal{E}}_{2}(1)\right\}$, $\operatorname{Pr}\left\{\overline{\mathcal{E}}_{3}(1,1)\right\}, \operatorname{Pr}\left\{\overline{\mathcal{E}}_{4}(1,1,1,1)\right\}$, and $\operatorname{Pr}\left\{\overline{\mathcal{E}}_{5}(1,1)\right\}$. We examine the remaining error probabilities. We obtain for $k \neq 1$

$$
\begin{aligned}
& \operatorname{Pr}\left\{\mathcal{E}_{1}(k)\right\} \\
& =\sum_{\substack{\left.u^{N}, v_{1}^{N}, v_{2}^{N}, x_{1}^{N}, z_{1}^{N}\right) \in \mathcal{T}_{\epsilon}^{N}}} P_{U V_{1} V_{2} X_{1} Z_{1}}\left(u^{N} v_{1}^{N} v_{2}^{N} x_{1}^{N} z_{1}^{N}\right) \\
& \stackrel{(a)}{=} \sum_{\substack{\left(u^{N}, v_{1}^{N}, v_{2}^{N}, x_{1}^{N}, z_{1}^{N}\right) \in \mathcal{T}_{\epsilon}^{N}}} P_{U V_{1} V_{2} X_{1}}\left(u^{N} v_{1}^{N} v_{2}^{N} x_{1}^{N}\right) P_{Z_{1} \mid U V_{1} X_{1}}\left(z_{1}^{N} \mid u^{N} v_{1}^{N} x_{1}^{N}\right) \\
& \stackrel{(b)}{\leq} 2^{N\left(H\left(U V_{1} V_{2} X_{1} Z_{1}\right)+\delta\right)} 2^{-N\left(H\left(U V_{1} V_{2} X_{1}\right)-\delta\right)} 2^{-N\left(H\left(Z_{1} \mid U V_{1} X_{1}\right)-\delta\right)} \\
& \leq 2^{-N\left(I\left(V_{2} ; Z_{1} \mid U V_{1} X_{1}\right)-\delta\right)} \\
& \stackrel{(c)}{=} 2^{-N\left(I\left(V_{2} ; Z_{1} \mid U X_{1}\right)-\delta\right)}
\end{aligned}
$$

where (a) follows from the Markov chain $V_{2}-U V_{1} X_{1}-$ $Z_{1}$ for $k \neq 1$; (b) follows by noticing that the typical set has a cardinality $2^{N\left(H\left(U V_{1} V_{2} X_{1} Z_{1}\right)+\delta\right)}$ and applying the joint

$$
\begin{aligned}
& \mathcal{E}_{1}\left(k_{b}^{\prime}\right)=\left\{\left(u^{N}\left(j_{b-1}, k_{b-1}\right), v_{1}^{N}\left(j_{b-1}, k_{b-1}, j_{b}^{\prime}\right), v_{2}^{N}\left(j_{b-1}, k_{b-1}, k_{b}^{\prime}\right), x_{1}^{N}\left(j_{b-1}, k_{b-1}, j_{b}^{\prime}, l_{b}\right), z_{1}^{N}(b)\right) \in \mathcal{T}_{\epsilon}^{N}\right\} \\
& \mathcal{E}_{2}\left(j_{b}^{\prime}\right)=\left\{\left(u^{N}\left(j_{b-1}, k_{b-1}\right), v_{1}^{N}\left(j_{b-1}, k_{b-1}, j_{b}^{\prime}\right), v_{2}^{N}\left(j_{b-1}, k_{b-1}, k_{b}^{\prime}\right), x_{2}^{N}\left(j_{b-1}, k_{b-1}, k_{b}^{\prime}, m_{b}\right), z_{2}^{N}(b)\right) \in \mathcal{T}_{\epsilon}^{N}\right\} \\
& \mathcal{E}_{3}\left(j_{B-1}, k_{B-1}\right) \\
& =\left\{\left(u^{N}\left(j_{B-1}, k_{B-1}\right), v_{1}^{N}\left(j_{B-1}, k_{B-1}, 1\right), v_{2}^{N}\left(j_{B-1}, k_{B-1}, 1\right), x_{1}^{N}\left(j_{B-1}, k_{B-1}, 1,1\right), x_{2}^{N}\left(j_{B-1}, k_{B-1}, 1,1\right), \tilde{y}^{N}(B)\right) \in \mathcal{T}_{\epsilon}^{N}\right\} \\
& \mathcal{E}_{4}\left(j_{b-1}, k_{b-1}, l_{b}, m_{b}\right) \\
& =\left\{\left(u^{N}\left(j_{b-1}, k_{b-1}\right), v_{1}^{N}\left(j_{b-1}, k_{b-1}, j_{b}^{\prime}\right), v_{2}^{N}\left(j_{b-1}, k_{b-1}, k_{b}^{\prime}\right), x_{1}^{N}\left(j_{b-1}, k_{b-1}, j_{b}^{\prime}, l_{b}\right), x_{2}^{N}\left(j_{b-1}, k_{b-1}, k_{b}^{\prime}, m_{b}\right), \tilde{y}^{N}(b)\right) \in \mathcal{T}_{\epsilon}^{N}\right\} \\
& \mathcal{E}_{5}\left(l_{1}, m_{1}\right)=\left\{\left(u^{N}(1,1), v_{1}^{N}\left(1,1, j_{b}^{\prime}\right), v_{2}^{N}\left(1,1, k_{b}^{\prime}\right), x_{1}^{N}\left(1,1, j_{b}^{\prime}, l_{b}\right), x_{2}^{N}\left(1,1, k_{b}^{\prime}, m_{b}\right), \tilde{y}^{N}(b)\right) \in \mathcal{T}_{\epsilon}^{N}\right\}
\end{aligned}
$$


typicality lemma [8, Chapter 2]; (c) follows from the Markov chain $V_{1}-U-V_{2}$. Following similar steps, we can prove

$$
\begin{aligned}
& \left.\operatorname{Pr}\left\{\mathcal{E}_{2}(j)\right)\right\} \leq 2^{-N\left(I\left(V_{1} ; Z_{2} \mid U X_{2}\right)-\delta\right)}, \quad \forall j \neq 1 \\
& \operatorname{Pr}\left\{\mathcal{E}_{3}(j, k)\right\} \leq 2^{-N\left(I\left(X_{1} X_{2} ; Y \mid S\right)-\delta\right)} \quad \forall(j, k) \neq(1,1) \\
& \operatorname{Pr}\left\{\mathcal{E}_{4}(j, k, l, m)\right\} \leq 2^{-N\left(I\left(X_{1} X_{2} ; Y \mid S\right)-\delta\right)}, \quad \forall(j, k) \neq(1,1), \forall(l, m) \\
& \operatorname{Pr}\left\{\mathcal{E}_{4}(1,1, l, m)\right\} \\
& =\operatorname{Pr}\left\{\mathcal{E}_{5}(l, m)\right\} \leq 2^{-N\left(I\left(X_{1} X_{2} ; Y \mid U V_{1} V_{2} S\right)-\delta\right)}, \quad \forall l \neq 1, m \neq 1 \\
& \operatorname{Pr}\left\{\mathcal{E}_{4}(1,1, l, 1)\right\} \\
& =\operatorname{Pr}\left\{\mathcal{E}_{5}(l, 1)\right\} \leq 2^{-N\left(I\left(X_{1} ; Y \mid U V_{1} X_{2} S\right)-\delta\right)}, \quad \forall l \neq 1 \\
& \operatorname{Pr}\left\{\mathcal{E}_{4}(1,1,1, m)\right\} \\
& =\operatorname{Pr}\left\{\mathcal{E}_{5}(1, m)\right\} \leq 2^{-N\left(I\left(X_{2} ; Y \mid U V_{2} X_{1} S\right)-\delta\right)}, \quad \forall m \neq 1
\end{aligned}
$$

Inserting (48) and (49) into (47), we have

$$
\begin{aligned}
P_{e}^{(n)} \leq & (B-1)\left(2^{N \cdot R_{12}} \cdot 2^{-N \cdot I\left(V_{1} ; Z_{2} \mid X_{2} U\right)}\right. \\
& \left.+2^{N \cdot R_{21}} \cdot 2^{-N \cdot I\left(V_{2} ; Z_{1} \mid X_{1} U\right)}\right) \\
& +2^{N\left(R_{12}+R_{21}\right)}+2^{-N \cdot I\left(X_{1} X_{2} ; Y \mid S\right)} \\
& +(B-2)\left(2^{N\left(R_{12}+R_{21}+R_{11}+R_{22}\right)} \cdot 2^{-N \cdot I\left(X_{1} X_{2} ; Y \mid S\right)}\right. \\
& +2^{N\left(R_{11}+R_{22}\right)} \cdot 2^{-N \cdot I\left(X_{1} X_{2} ; Y \mid S V_{1} V_{2} U\right)} \\
& +2^{N \cdot R_{11}} \cdot 2^{-N \cdot I\left(X_{1} ; Y \mid S X_{2} V_{1} U\right)} \\
& \left.+2^{N \cdot R_{22}} \cdot 2^{-N \cdot I\left(X_{2} ; Y \mid S X_{1} V_{2} U\right)}\right) \\
& +2^{N\left(R_{11}+R_{22}\right)} \cdot 2^{-N \cdot I\left(X_{1} X_{2} ; Y \mid S, V_{1} V_{2} U\right)} \\
& +2^{N \cdot R_{11}} \cdot 2^{-N \cdot I\left(X_{1} ; Y \mid S X_{2} V_{1} U\right)} \\
& +2^{N \cdot R_{22}} \cdot 2^{-N \cdot I\left(X_{2} ; Y \mid S X_{1} V_{2} U\right)}+\epsilon_{N}^{\prime}
\end{aligned}
$$

where $\epsilon_{N}^{\prime}$ denotes a constant which vanishes as $N$ grows.

\section{APPENDIX D}

\section{AnAlysis of AVERAGE Distortion}

For a given message pair $\left(w_{1}, w_{2}\right)$, we bound the average distortion for encoder 1 .

$$
\begin{aligned}
& d_{1}^{(n)}\left(w_{1}, w_{2}\right) \\
& =\mathbb{E}\left[\mathbb{E}\left[\frac{1}{n} \sum_{n=1}^{N} d_{1}\left(S_{1 i}, \hat{S}_{1 i}\right) \mid X_{1}^{n}\left(w_{1}, Z_{1}^{n}\right), V_{2}^{n}\left(w_{2}, Z_{2}^{n}\right)\right]\right] \\
& \stackrel{(a)}{\leq} P_{e}^{(n)}\left(w_{1}, w_{2}\right) d_{\max }+\left(1-P_{e}^{(n)}\left(w_{1}, w_{2}\right)\right)(1+\epsilon) \\
& \frac{1}{n} \sum_{i=1}^{n} \mathbb{E}\left[\mathbb{E}\left[d_{1}\left(S_{1 i}, \underline{\psi}_{1}^{*}\left(x_{1}, v_{2}, Z_{1 i}\right)\right) \mid X_{1 i}=x_{1}, V_{2 i}=v_{2}\right]\right] \\
& \stackrel{(b)}{=} P_{e}^{(n)}\left(w_{1}, w_{2}\right) d_{\max } \\
& \quad+\left(1-P_{e}^{(n)}\left(w_{1}, w_{2}\right)\right)(1+\epsilon) \mathbb{E}\left[\underline{c}_{1}\left(X_{1}, V_{2}\right)\right] .
\end{aligned}
$$

where (a) follows by applying the upper bound on the distortion function to the decoding error event and the typical average lemma [Ch. 2.4] [8] to the successful decoding event; (b) follows from the cost function applied to each time $i$. Now, we average over all possible message pairs and obtain the average distortion for encoder 1 as:

$$
\begin{aligned}
d_{1}^{(n)} & =\frac{1}{\left|\mathcal{W}_{1}\right|\left|\mathcal{W}_{2}\right|} \sum_{\left(w_{1}, w_{2}\right) \in \mathcal{W}_{1} \times \mathcal{W}_{2}} d_{1}^{(n)}\left(w_{1}, w_{2}\right) \\
& \leq \frac{\left(1-P_{e}^{(n)}\right)(1+\epsilon)}{\left|\mathcal{W}_{1}\right|\left|\mathcal{W}_{2}\right|} \sum_{\left(w_{1}, w_{2}\right) \in \mathcal{W}_{1} \times \mathcal{W}_{2}} \mathbb{E}\left[\underline{c}_{1}\left(X_{1}\left(w_{1}\right), V_{2}\left(w_{2}\right)\right)\right] \\
& +d_{\max } P_{e}^{(n)} \\
& =\frac{\left(1-P_{e}^{(n)}\right)(1+\epsilon)}{n} \sum_{i=1}^{n} \sum_{\left(x_{1}, v_{2}\right)} P_{X_{1 i}, V_{2 i}}\left(x_{1}, v_{2}\right) \underline{c}_{1}\left(x_{1 i}, v_{2 i}\right) \\
& +d_{\max } P_{e}^{(n)}
\end{aligned}
$$

where the last equality follows from the uniformly distributed message pair such that we have $\left(X_{1 i}\left(w_{1}\right), V_{2 i}\left(w_{2}\right)\right)$ with probability $\frac{1}{\left|\mathcal{W}_{1} \| \mathcal{W}_{2}\right|}$ for every pair $\left(w_{1}, w_{2}\right) \in \mathcal{W}_{1} \times \mathcal{W}_{2}$ Therefore, it readily follows

$$
\limsup _{n \rightarrow \infty} d_{1}^{(n)} \leq \sum_{\left(x_{1}, v_{2}\right)} P_{X_{1} V_{2}}\left(x_{1}, v_{2}\right) \underline{c}_{1}\left(x_{1}, v_{2}\right) \leq D_{1} .
$$

Similarly, we obtain also the desired result for encoder 2 .

\section{APPENDIX E}

\section{Calculation of Optimal Estimate Cost (18)}

We consider user 1 and provide the estimator $\psi_{1}^{*}\left(x_{1}, x_{2}, y\right)$ as the solution of

$$
\begin{array}{r}
\arg \min _{\psi}\left[P_{S_{1} \mid X_{1}, X_{2}, Y}\left(0 \mid x_{1}, x_{2}, y\right)\left(0 \oplus \psi\left(x_{1}, x_{2}, y\right)\right)\right. \\
\left.+P_{S_{1} \mid X_{1}, X_{2}, Y}\left(1 \mid x_{1}, x_{2}, y\right)\left(1 \oplus \psi\left(x_{1}, x_{2}, y\right)\right)\right]
\end{array}
$$

and let $d_{1}\left(x_{1}, x_{2}, y\right)$ denote the resulting value. Then, the cost function is given by

$$
c_{1}\left(x_{1}, x_{2}\right)=\sum_{y} P_{Y}(y) d_{1}\left(x_{1}, x_{2}, y\right)
$$

We have two simple estimators. In the first case, the state can be estimated perfectly yielding $d_{1}\left(x_{1}, x_{2}, y\right)=0$. This case includes:

$$
\begin{aligned}
& \psi_{1}^{*}(1,0,0)=0, \quad \psi_{1}^{*}(1,0,1)=1 \\
& \psi_{1}^{*}(1,1,0)=0, \quad \psi_{1}^{*}(1,1,2)=1
\end{aligned}
$$

In the second case, the state of interest is erased either by the input symbol $x_{1}$ or the erasure event $s_{1}+s_{2}=1$. Then, we choose a fixed estimator given by

$$
\begin{aligned}
\psi_{1}^{*}\left(x_{1}, x_{2}, y\right) & =\arg \min _{\psi \in\{0,1\}}\left[\bar{p}_{s}(0 \oplus \psi)+p_{s}(1 \oplus \psi)\right] \\
& = \begin{cases}0, & \text { if } p_{s}<\frac{1}{2} \\
1, & \text { else }\end{cases}
\end{aligned}
$$

This fixed estimator yields $d_{1}\left(x_{1}, x_{2}, y\right)=\eta=\min \left\{p_{s}, 1-\right.$ $\left.p_{s}\right\}$ for

$$
\left(x_{1}, x_{2}, y\right) \in\{(0,0,0),(0,1,0),(0,1,1),(1,1,1)\}
$$

Plugging the results of (54) and (56) into (53), we obtain the desired result. 


\section{APPENDIX F}

Calculation of Achievable Cost (23)

We provide the estimator $\underline{\psi}_{1}^{*}\left(x_{1}, v_{2}, y\right)$ as the solution of

$$
\begin{array}{r}
\arg \min _{\psi}\left[P_{S_{1} \mid X_{1}, V_{2}, Y}\left(0 \mid x_{1}, v_{2}, y\right)\left(0 \oplus \psi\left(x_{1}, v_{2}, y\right)\right)\right. \\
\left.+P_{S_{1} \mid X_{1}, V_{2}, Y}\left(1 \mid x_{1}, v_{2}, y\right)\left(1 \oplus \psi\left(x_{1}, v_{2}, y\right)\right)\right]
\end{array}
$$

and let $\underline{d}_{1}\left(x_{1}, v_{2}, y\right)$ denote the resulting value. Then, the cost function is given by

$$
\underline{c}_{1}\left(x_{1}, v_{2}\right)=\sum_{y} P_{Y}(y) \underline{d}_{1}\left(x_{1}, v_{2}, y\right)
$$

We have three cases. In the first case, the state can be estimated perfectly by achieving $\underline{d}_{1}\left(x_{1}, v_{2}, y\right)=0$. We have:

$$
\begin{aligned}
& \underline{\psi}_{1}^{*}(1,0,0)=0, \quad \underline{\psi}_{1}^{*}(1,0,2)=1 \\
& \underline{\psi}_{1}^{*}(1,1,0)=0, \quad \underline{\psi}_{1}^{*}(1,1,2)=1
\end{aligned}
$$

In the second case, $s_{1}$ is erased by the associated input symbol, i.e. $x_{1}$ for encoder 1 . Then, we choose a fixed estimator independent of $\left(v_{2}, y\right)$ as in (59). This case includes:

$$
\left(x_{1}, v_{2}, y\right) \in\{(0,0,0),(0,1,0),(0,1,1)\}
$$

yielding $\underline{d}_{1}\left(x_{1}, v_{2}, y\right)=\eta=\min \left\{p_{s}, 1-p_{s}\right\}$. In the last case $s_{1}$ cannot be correctly estimated due to the interference caused by $s_{2} \theta_{2}$. Noticing $P_{S_{1} \mid X_{1} V_{2} Y}\left(s_{1} \mid 1,0,1\right)=p_{s} r$ if $s_{1}=0$ and $P_{S_{1} \mid X_{1} V_{2} Y}\left(s_{1} \mid 1,1,1\right)=p_{s} \bar{r}$ if $s_{1}=0$ we have

$$
\begin{aligned}
\underline{\psi}_{1}^{*}(1,0,1) & =\arg \min _{\psi \in\{0,1\}}\left[p_{s} r(0 \oplus \psi)+\left(1-p_{s} r\right)(1 \oplus \psi)\right] \\
& = \begin{cases}1, & \text { if } p_{s} r<\frac{1}{2} \\
0, & \text { else }\end{cases}
\end{aligned}
$$

yielding $\underline{d}_{1}(1,0,1)=\min \left\{p_{s} r, 1-p_{s} r\right\}=\eta_{r}$. Similarly we have $\underline{d}_{1}(0,0,1)=\min \left\{p_{s} r, 1-p_{s} r\right\}=\eta_{r}$ and $\underline{d}_{1}(1,1,1)=$ $\min \left\{p_{s} \bar{r}, 1-p_{s} \bar{r}\right\}=\eta_{\bar{r}}$. By combining three cases and using (57), we obtain the desired result.

\section{APPENDIX G}

\section{PROOF OF COROLLARY 1}

Applying Theorem 2 for the erasure MAC with binary states, we have

$$
\begin{aligned}
R_{\text {sum-prop }}(\infty) & \leq \min \left\{H(Y \mid S), H\left(Y \mid S, V_{1}, V_{2}, U\right)\right. \\
& \left.+2\left\{H\left(Y \mid X_{2}, U\right)-H\left(Y \mid X_{2}, U, V_{1}\right)\right\}\right\}
\end{aligned}
$$

In order to characterize each term inside min, we first provide the input and output distribution. We have

$$
\begin{aligned}
& P_{X_{1}, X_{2}}(0,0)=\bar{p} \kappa^{2}+p \bar{\kappa}^{2}, \quad P_{X_{1}, X_{2}}(1,1)=p \kappa^{2}+\bar{p} \bar{\kappa}^{2} \\
& P_{X_{1}, X_{2}}(0,1)=P_{X_{1}, X_{2}}(1,0)=\kappa \bar{\kappa}
\end{aligned}
$$

as well as

$$
\begin{aligned}
& P_{Y}(0)=P_{X_{1}, X_{2}}(0,0)+2 \bar{p}_{s} P_{X_{1}, X_{2}}(0,1)+\bar{p}_{s}^{2} P_{X_{1}, X_{2}}(1,1) \\
& P_{Y}(1)=2 p_{s} P_{X_{1}, X_{2}}(0,1)+2 p_{s} \bar{p}_{s} P_{X_{1}, X_{2}}(1,1) \\
& P_{Y}(2)=p_{s}^{2} P_{X_{1}, X_{2}}(1,1)
\end{aligned}
$$

Now we will examine each term inside min.

Term $f_{2}(p, q, r)=H(Y \mid S)$

$$
f_{1}(p, q, r)=-\sum_{\left(s_{1}, s_{2}\right)} P_{S_{1}}\left(s_{1}\right) P_{S_{2}}\left(s_{2}\right) \sum_{y} P_{Y \mid S_{1} S_{2}}\left(y \mid s_{1}, s_{2}\right)
$$$$
\log P_{Y \mid S_{1} S_{2}}\left(y \mid s_{1}, s_{2}\right)
$$

where

$$
\begin{aligned}
& P_{Y \mid S_{1} S_{2}}(0 \mid 0,0)=1, P_{Y \mid S_{1} S_{2}}(y \mid 0,0)=0, \forall y \in\{1,2\} \\
& P_{Y \mid S_{1} S_{2}}(0 \mid 0,1)=P_{X_{2}}(0)=\bar{p} \kappa^{2}+p \bar{\kappa}^{2}+\kappa \bar{\kappa} \\
& P_{Y \mid S_{1} S_{2}}(1 \mid 0,1)=P_{X_{2}}(1)=\kappa \bar{\kappa}+p \kappa^{2}+\bar{p} \bar{\kappa}^{2} \\
& P_{Y \mid S_{1} S_{2}}(0 \mid 1,1)=P_{X_{1} X_{2}}(0,0)=\bar{p} \kappa^{2}+p \bar{\kappa}^{2} \\
& P_{Y \mid S_{1} S_{2}}(1 \mid 1,1)=P_{X_{1} X_{2}}(0,1)+P_{X_{1} X_{2}}(1,0)=2 \kappa \bar{\kappa} \\
& P_{Y \mid S_{1} S_{2}}(2 \mid 1,1)=P_{X_{1} X_{2}}(1,1)=p \kappa^{2}+\bar{p} \bar{\kappa}^{2}
\end{aligned}
$$

Plugging these expressions into (63), we obtain $f_{2}=2 p_{s} \bar{p}_{s} H_{2}(p \kappa+\bar{p} \bar{\kappa})+p_{s}^{2} H_{3}\left(\bar{p} \kappa^{2}+p \bar{\kappa}^{2}, 2 \kappa \bar{\kappa}, p \kappa^{2}+\bar{p} \bar{\kappa}^{2}\right)$

Term $f_{1 a}=H\left(Y \mid S, V_{1}, V_{2}, U\right)$

By defining

$$
Y^{\prime}=S_{1} \Theta_{1}+S_{2} \Theta_{2}
$$

we have

$$
\begin{aligned}
& H\left(Y \mid S, V_{1}, V_{2}, U\right)=H\left(Y^{\prime} \mid S\right) \\
& =\sum_{\left(s_{1} s_{2}\right)} P_{S_{1} S_{2}}\left(s_{1}, s_{2}\right) \sum_{y^{\prime}} P_{Y^{\prime} \mid S}\left(y^{\prime} \mid s\right) \log P_{Y^{\prime} \mid S}\left(y^{\prime} \mid s\right)
\end{aligned}
$$

where we have

$P_{Y^{\prime} \mid S}\left(y^{\prime} \mid 00\right)=\mathbf{1}\left\{y^{\prime}=0\right\}$

$P_{Y^{\prime} \mid S}\left(y^{\prime} \mid 01\right)=\bar{r} \mathbf{1}\left\{y^{\prime}=0\right\}+r \mathbf{1}\left\{y^{\prime}=1\right\}$

$P_{Y^{\prime} \mid S}\left(y^{\prime} \mid 01\right)=\bar{r} \mathbf{1}\left\{y^{\prime}=0\right\}+r \mathbf{1}\left\{y^{\prime}=1\right\}$

$P_{Y^{\prime} \mid S}\left(y^{\prime} \mid 11\right)=\bar{r}^{2} \mathbf{1}\left\{y^{\prime}=0\right\}+2 r \bar{r} \mathbf{1}\left\{y^{\prime}=1\right\}+r^{2} \mathbf{1}\left\{y^{\prime}=2\right\}$

yielding

$$
f_{1 a}=2 \bar{p}_{s} p_{s} H_{2}(r)+p_{s}^{2} H_{3}\left(r^{2}, 2 r \bar{r}, \bar{r}^{2}\right) .
$$

Term $f_{1 b}=H\left(Y \mid X_{2}, U\right)$

$$
\begin{aligned}
H\left(Y \mid X_{2}, U\right)= & \sum_{u} P_{U}(u) \sum_{x_{2}} P_{X_{2} \mid U}\left(x_{2} \mid u\right) \\
& \sum_{y} P_{Y \mid X_{2} U}\left(y \mid x_{2} u\right) \log P_{Y \mid X_{2} U}\left(y \mid x_{2} u\right)
\end{aligned}
$$

with

$$
\begin{aligned}
P_{Y \mid X_{2}, U}\left(y \mid x_{2}, u\right) & =\sum_{s} P_{S}(s) P_{Y \mid S, X_{2}, U}\left(y \mid s, x_{2}, u\right) \\
& =\bar{p}_{s}^{2} \mathbf{1}\left\{y=0, \forall\left(x_{2}, u\right)\right\} \\
& \left.+\bar{p}_{s} p_{s} \mathbf{1}\left\{y=x_{2}, \forall u\right)\right\} \\
& +p_{s} \bar{p}_{s} P_{X_{1} \mid U}(y \mid u) \mathbf{1}\{y \in\{0,1\}\} \\
& +p_{s}^{2} P_{X_{1} \mid U}\left(y-x_{2} \mid u\right) \mathbf{1}\left\{\left(y-x_{2}\right) \in\{0,1\}\right\}
\end{aligned}
$$


yielding

$$
\begin{aligned}
& P_{Y \mid X_{2}, U}(0 \mid 0,0)=\bar{p}_{s}+p_{s} \kappa \\
& P_{Y \mid X_{2}, U}(1 \mid 0,0)=p_{s} \bar{\kappa} \\
& P_{Y \mid X_{2}, U}(2 \mid 0,0)=0 \\
& P_{Y \mid X_{2}, U}(0 \mid 0,1)=\bar{p}_{s}+p_{s} \bar{\kappa} \\
& P_{Y \mid X_{2}, U}(1 \mid 0,1)=p_{s} \kappa \\
& P_{Y \mid X_{2}, U}(2 \mid 0,1)=0 \\
& P_{Y \mid X_{2}, U}(0 \mid 1,0)=\bar{p}_{s}^{2}+p_{s} \bar{p}_{s} \kappa \\
& P_{Y \mid X_{2}, U}(1 \mid 1,0)=\bar{p}_{s} p_{s}+p_{s} \bar{p}_{s} \bar{\kappa}+p_{s}^{2} \kappa \\
& P_{Y \mid X_{2}, U}(2 \mid 1,0)=p_{s}^{2} \bar{\kappa} \\
& P_{Y \mid X_{2}, U}(0 \mid 1,1)=\bar{p}_{s}^{2}+p_{s} \bar{p}_{s} \bar{\kappa} \\
& P_{Y \mid X_{2}, U}(1 \mid 1,1)=\bar{p}_{s} p_{s}+p_{s} \bar{p}_{s} \kappa+p_{s}^{2} \bar{\kappa} \\
& P_{Y \mid X_{2}, U}(2 \mid 1,1)=p_{s}^{2} \kappa
\end{aligned}
$$

where we used

$$
\begin{aligned}
& P_{X_{1} \mid U}(0 \mid 0)=P_{X_{1} \mid U}(1 \mid 1)=\kappa \\
& P_{X_{1} \mid U}(0 \mid 1)=P_{X_{1} \mid U}(1 \mid 0)=\bar{\kappa}
\end{aligned}
$$

Plugging (69) into (67) and using

$$
\begin{aligned}
& P_{X_{2} U}(0,0)=P_{U}(0) P_{X_{2} \mid U}(0 \mid 0)=\bar{p} \kappa \\
& P_{X_{2} U}(0,1)=P_{U}(1) P_{X_{2} \mid U}(0 \mid 1)=p \bar{\kappa} \\
& P_{X_{2} U}(1,0)=P_{U}(0) P_{X_{2} \mid U}(1 \mid 0)=\bar{p} \bar{\kappa} \\
& P_{X_{2} U}(1,1)=P_{U}(1) P_{X_{2} \mid U}(1 \mid 1)=p \kappa
\end{aligned}
$$

we obtain the desired expression for $f_{1 b}$.

Term : $f_{1 c}=H\left(Y \mid U, V_{1}, X_{2}\right)$

We have

$$
\begin{aligned}
& H\left(Y \mid U, V_{1}, X_{2}\right)=\sum_{u} P_{U}(u) \sum_{x_{2}} P_{X_{2} \mid U}\left(x_{2} \mid u\right) \sum_{v_{1}} P_{V_{1} \mid U}\left(v_{1} \mid u\right) \\
& \sum_{y} P_{Y \mid U V_{1} X_{2}}\left(y \mid u v_{1} x_{2}\right) \log P_{Y \mid U V_{1} X_{2}}\left(y \mid u v_{1} x_{2}\right)
\end{aligned}
$$

where

$$
\begin{aligned}
& P_{Y \mid U, V_{1}, X_{2}}\left(y \mid u, v_{1}, x_{2}\right)=P_{Y \mid V_{1}, X_{2}}\left(y \mid v_{1}, x_{2}\right) \\
& =\sum_{s} P_{S}(s) P_{Y \mid S, V_{1}, X_{2}}\left(y \mid s, v_{1}, x_{2}\right) \\
& =\bar{p}_{s}^{2} \mathbf{1}\left\{y=0, \forall\left(x_{2} v_{1}\right)\right\}+p_{s} \bar{p}_{s} \mathbf{1}\left\{y=x_{2}, \forall v_{1}\right\} \\
& +p_{s} \bar{p}_{s} P_{\Theta_{1}}\left(y \oplus v_{1}\right) \mathbf{1}\left\{y \in\{0,1\}, \forall x_{2}\right\} \\
& +p_{s}^{2} P_{\Theta_{1}}\left(\left(y-x_{2}\right) \oplus v_{1}\right) \mathbf{1}\left\{\left(y-x_{2}\right) \in\{0,1\}\right\}
\end{aligned}
$$

yielding

$$
\begin{aligned}
& P_{Y \mid V_{1}, X_{2}}(0 \mid 0,0)=\bar{p}_{s}+p_{s} \bar{r} \\
& P_{Y \mid V_{1}, X_{2}}(1 \mid 0,0)=p_{s} r \\
& P_{Y \mid V_{1}, X_{2}}(2 \mid 0,0)=0 \\
& P_{Y \mid V_{1}, X_{2}}(0 \mid 0,1)=\bar{p}_{s}^{2}+p_{s} \bar{p}_{s} \bar{r} \\
& P_{Y \mid V_{1}, X_{2}}(1 \mid 0,1)=p_{s} \bar{p}_{s}+p_{s} \bar{p}_{s} r+p_{s}^{2} \bar{r} \\
& P_{Y \mid V_{1}, X_{2}}(2 \mid 0,1)=p_{s}^{2} r
\end{aligned}
$$

$$
\begin{aligned}
& P_{Y \mid V_{1}, X_{2}}(0 \mid 1,0)=\bar{p}_{s}+p_{s} r \\
& P_{Y \mid V_{1}, X_{2}}(1 \mid 1,0)=p_{s} \bar{r} \\
& P_{Y \mid V_{1}, X_{2}}(2 \mid 1,0)=0 \\
& P_{Y \mid V_{1}, X_{2}}(0 \mid 1,1)=\bar{p}_{s}^{2}+p_{s} \bar{p}_{s} r \\
& P_{Y \mid V_{1}, X_{2}}(1 \mid 1,1)=p_{s} \bar{p}_{s}+p_{s} \bar{p}_{s} \bar{r}+p_{s}^{2} r \\
& P_{Y \mid V_{1}, X_{2}}(2 \mid 1,1)=p_{s}^{2} \bar{r}
\end{aligned}
$$

By noticing

$$
\begin{aligned}
P_{V_{1} X_{2}}\left(v_{1}, x_{2}\right) & =\sum_{u} P_{U}(u) P_{V_{1} \mid U}\left(v_{1} \mid u\right) P_{X_{2} \mid U}\left(x_{2} \mid u\right) \\
& =\bar{p} P_{\Sigma_{1}}\left(v_{1}\right) P_{X_{2} \mid U}\left(x_{2} \mid 0\right)+p P_{\Sigma_{1}}\left(\bar{v}_{1}\right) P_{X_{2} \mid U}\left(x_{2} \mid 1\right)
\end{aligned}
$$

we readily obtain

$$
\begin{aligned}
& P_{V_{1} X_{2}}(0,0)=\bar{p} \bar{q} \kappa+p q \bar{\kappa} \\
& P_{V_{1} X_{2}}(0,1)=\bar{p} \bar{q} \bar{\kappa}+p q \kappa \\
& P_{V_{1} X_{2}}(1,0)=\bar{p} q \kappa+p \bar{q} \bar{\kappa} \\
& P_{V_{1} X_{2}}(1,1)=\bar{p} q \bar{\kappa}+p \bar{q} \kappa
\end{aligned}
$$

Plugging these expressions into (72), we obtain the desired result. 